\title{
Robust Stackelberg controllability for the Navier-Stokes equations
}

\author{
Cristhian Montoya and Luz de Teresa*
}

\begin{abstract}
In this paper we deal with a robust Stackelberg strategy for the Navier-Stokes system. The scheme is based in considering a robust control problem for the "follower control" and its associated disturbance function. Afterwards, we consider the notion of Stackelberg optimization (which is associated to the "leader control") in order to deduce a local null controllability result for the Navier-Stokes system.

Key Words: Robust control, hierarchic control, Navier-Stokes equations, Carleman estimates MSC 35Q30, 35Q93, 49J20, 91A65, 93C10
\end{abstract}

\section{Introduction}

The theory of robust control began in the late 1970s and early 1980s for finite dimensional systems. Since then, many techniques have been developed to deal with systems with uncertainties. In the late 90s the papers of Bewley et. al [5] presented the first rigorous generalization of the concepts in the case of partial differential equations. What could we understand by robustness in a control system? Well, informally, a controller designed for a particular set of parameters is said to be robust if it also functions correctly under a uncertainty: the controller is designed to work assuming that certain variable will be unknown. In this sense, one could think in the worst-case disturbance of the system, and design a controller which is suited to handle even this extreme situation. Thus, the problem of finding a robust control involves the problem of finding the worst-case disturbance in the spirit of a non-cooperative game (when there is not cooperation between the controller and disturbance function), which is from the mathematical point of view to reach a saddle point for the pair disturbance-controller.

The research on robust control for PDE systems is in an early stage. Much of the literature deals with numerical aspects and much of the theory has been developed for fluid mechanics and for some elliptic problems. See e.g. [3, 5, 4, 20, 17]. In this paper we will present a hierarchic strategy to deal with robust control and, simultaneously, with null control for incompressible fluids modelled by the Navier-Stokes equations with Dirichlet boundary conditions.

We will work in the setting of a Stackelberg competition, see [22]. This consists in a noncooperative decision problem in which one of the participants enforce its strategy on the other participants. We assume that we can act on the dynamics of the system through a hierarchy of controls. In our case the controls are external forces acting on the system, where the leader control has a local null controllability objective while the follower control and perturbation solve a robust control problem.

To be precise: let $\Omega$ be a nonempty bounded connected open subset of $\mathbb{R}^{N}(N=2$ or $N=3)$ of class $C^{\infty}$. Let $T>0$ and let $\omega$ and $\mathcal{O}$ be (small) nonempty open subsets of $\Omega$ with $\omega \cap \mathcal{O}=\emptyset$. We will use the notation $Q:=\Omega \times(0, T), \Sigma:=\partial \Omega \times(0, T)$ and $n(x)$ will denote the outward

\footnotetext{
*Universidad Nacional Autónoma de México, UNAM, Instituto de Matemáticas, Ciudad de México, México. emails: cmontoya@matem.unam.mx, ldeteresa@im.unam.mx. The research was partially supported by UNAMDGAPA-PAPIIT IN102116. The first author is supported by project FORDECYT 265667 of CONACYT
} 
unit normal vector at the point $x \in \partial \Omega$.

Let us consider the Navier-Stokes system with homogeneous Dirichlet boundary conditions

$$
\begin{cases}y_{t}-\Delta y+(y \cdot \nabla) y+\nabla p=h 1_{\omega}+v \chi_{\mathcal{O}}+\psi & \text { in } Q \\ \nabla \cdot y=0 & \text { in } Q \\ y=0 & \text { on } \Sigma, \\ y(\cdot, 0)=y_{0}(\cdot) & \text { in } \Omega,\end{cases}
$$

where $h=h(x, t) \in L^{2}(\omega \times(0, T))^{N}$ is called the "leader control", $v=v(x, t) \in L^{2}(\mathcal{O} \times(0, T))^{N}$ is the "follower control", $\psi \in L^{2}(Q)^{N}$ is an unknown perturbation and $y_{0}$ an initial state in a suitable space. Here $1_{\omega}$ is the characteristic function of the set $\omega$ and $\chi_{\mathcal{O}}$ is a smooth nonnegative function such that $\operatorname{supp} \chi_{\mathcal{O}}=\overline{\mathcal{O}}$.

To our knowledge there are not results in the literature concerning a robust Stackelberg strategy for system (1.1). As far as we know, the first paper on robust Stackelberg controllability is [18], which develops the concept of control for a semi-linear parabolic equation. However, there exist several papers which treat independently robust and hierarchical control for the Navier-Stokes system. In the context of robust control (that is $h \equiv 0$ in (1.1)), the works [6] and [5] show the existence and uniqueness of the solution to the robust control problem for the $N$-dimensional case of system (1.1), and present an appropriate numerical method to solve it. In their works the authors have used an abstract scheme throughout Leray projection and classical techniques of optimal control theory. In [20], some theoretical and numerical aspects are presented for the optimal and robust control of the Navier-Stokes equations. Additional information on optimal and robust control theory for linear and nonlinear systems can be found in [4], [20], [13], and references therein.

In the context of hierarchical control (that is $\psi \equiv 0$ in (1.1)), some recent works such as $[12,1,2,19]$ and $[18]$ show a strategy with a leader and follower controls for different equations. Some older results on a Stackelberg-Nash control strategy were proved by J. Diaz and J-L. Lions in [7] for a linear parabolic problem and by J. Limaco et al. for a linear parabolic problem with moving boundaries [19]. In both cases, the objective of the leader control is an approximate controllability result. In the case of linear fluid models some approximate controllability of Stackelberg-Nash strategies started with the result of F. Guillén- González et. al for the Stokes system [12], and were extended by F. Araruna et al. for linearized micropolar fluids [1]. In [1] the main arguments are based on a Fenchel- Rockafeller dual variational principle [23]. For semilinear parabolic equations, a Stackelberg-Nash strategy with exact controllability for the leader control is proved in [2] using Carleman inequalities.

In our work, we follow the ideas introduced in [18] for the Navier-Stokes equations with Dirichlet boundary conditions. However the non linearity of (1.1) will allows only to obtain a local null controllability result for the leader control.

Let us now introduce the usual spaces in the context of incompressible fluids ([21]):

$$
\begin{aligned}
& H:=\left\{u \in L^{2}(\Omega)^{N}: \nabla \cdot u=0, \text { in } \Omega, u \cdot n=0 \text { on } \partial \Omega\right\}, \\
& V:=\left\{u \in H_{0}^{1}(\Omega)^{N}: \nabla \cdot u=0 \text { in } \Omega\right\} .
\end{aligned}
$$

Following the scheme for the robust control problem given in $[3,6]$, the general space for the control functions and the disturbance $\psi$ in the right-hand side of $(1.1)$ is $L^{2}(0, T ; H)$.

Now, we focus our attention on the control problem we are interested in.

\subsection{The main problem}

Given $h \in L^{2}(\omega \times(0, T))^{N}$ a (leader) control, we consider the secondary cost functional

$$
J_{r}(\psi, v ; h):=\frac{\mu}{2} \iint_{\mathcal{O}_{d} \times(0, T)}\left|y-y_{d}\right|^{2} d x d t+\frac{1}{2}\left(\ell^{2} \iint_{\mathcal{O} \times(0, T)} \chi_{\mathcal{O}}|v|^{2} d x d t-\gamma^{2} \iint_{Q}|\psi|^{2} d x d t\right),
$$


where $\ell, \gamma, \mu>0$ are constants, $\mathcal{O}_{d}$ is an open subset of $\Omega$, which represents a observability domain, and $y_{d} \in L^{2}\left(0, T ; L^{2}\left(\mathcal{O}_{d}\right)^{N}\right)$ is given. The constant $\mu$ arises from the physical parameters that govern the motion of fluids such as viscosity, characteristic length and characteristic velocity. The parameters $\ell, \gamma$ are included to make the cost functional consistent and to account the relative weight of each term. Note that the sign of the term associated to the disturbance is opposite to the sign used for the control, this is because we minimize with respect to the control $v$ meanwhile simultaneouly maximize with respect to the disturbance $\psi$. From another perspective, the term $-\gamma^{2}\|\psi\|_{L^{2}(Q)^{N}}^{2}$ constrains the magnitude of the disturbance function in the maximization with respect to $\psi$ and, the term associated to $\ell^{2}\|v\|_{L^{2}(Q)^{N}}^{2}$ constrains the magnitude of the control in the minimization with respect to $v$.

To explain the robust Stackelberg control problem, we will consider the following two subproblems:

i) First problem. For every fixed leader control $h$, solve the robust control problem for the nonlinear system (1.1), that is, find the best control $v$ in the presence of the disturbance $\psi$ which maximally spoils the follower control for the Navier-Stokes system (1.1). The robust control problem to be solved is given in the following definition.

Definition 1.1. Let $h \in L^{2}(\omega \times(0, T))^{N}$ be fixed. The disturbance $\bar{\psi} \in L^{2}(Q)^{N}$, the control $\bar{v} \in L^{2}(Q)^{N}$, and the solution $\bar{y}=y(h, \bar{v}(h), \bar{\psi}(h))$ of (1.1) associated with $(\bar{\psi}(h), \bar{v}(h))$ are said to solve the robust control problem when a saddle point $(\bar{\psi}(h), \bar{v}(h))$ of the cost functional defined in (1.2) is reached, that is, if

$$
J_{r}(\psi, \bar{v}(h) ; h) \leq J_{r}(\bar{\psi}(h), \bar{v}(h) ; h) \leq J_{r}(\bar{\psi}(h), v(h) ; h), \quad \forall(\psi, v) \in L^{2}(Q)^{N \times N} .
$$

In this case,

$$
J_{r}(\bar{\psi}(h), \bar{v}(h) ; h)=\max _{\psi \in L^{2}(Q)^{N}} \min _{v \in L^{2}(Q)^{N}} J_{r}(\psi, v ; h)=\min _{v \in L^{2}(Q)^{N}} \max _{\psi \in L^{2}(Q)^{N}} J_{r}(\psi, v ; h) .
$$

ii) Second problem. Once the saddle point has been identified for each leader control $h$, this is, once the existence of the saddle point $(\bar{\psi}(h), \bar{v}(h))$ for every leader control $h$ is guarantied, we deal with the problem of finding the control $h$ of minimal norm satisfying null controllability constraints. More precisely, we look for an optimal control $\bar{h}$ such that

$$
J(\bar{h})=\min _{h} \frac{1}{2} \iint_{\omega \times(0, T)}|h|^{2} d x d t, \quad \text { subject to the restriction } \quad y(\cdot, T)=0 \text { in } \Omega .
$$

Our main result on the robust hierarchic control is given in the following theorem.

Theorem 1.1. Assume that $\omega \cap \mathcal{O}_{d} \neq \emptyset$. Then, for every $T>0$ and $\mathcal{O}, \omega \subset \Omega$ open subsets such that $\mathcal{O} \cap \omega=\emptyset$, there exist $\gamma_{0}, \ell_{0}, \delta$ and a positive function $\rho=\rho(t)$ blowing up $t=T$ such that for any $\gamma \geq \gamma_{0}, \ell \geq \ell_{0}, y_{0} \in V$ and $y_{d} \in L^{2}\left(0, T ; L^{2}\left(\mathcal{O}_{d}\right)^{N}\right)$ satisfying

$$
\left\|y_{0}\right\|_{V} \leq \delta \quad \text { and } \quad \iint_{\mathcal{O}_{d} \times(0, T)} \rho^{2}(t)\left|y_{d}\right|^{2} d x d t<+\infty
$$

we can find a leader control $h \in L^{2}\left(0, T ; L^{2}(\omega)^{N}\right)$ and an unique saddle point $(\bar{\psi}, \bar{v})$ on $L^{2}(Q)^{N} \times$ $L^{2}\left(0, T ; L^{2}(\mathcal{O})^{N}\right)$ and an associated solution $(y, p)$ to $(1.1)$ verifying $y(\cdot, T)=0$ in $\Omega$.

In order to prove Theorem 1.1, we shall mainly consider two steps: a) the robust control results established in [6] allow us to solve the mentioned-above first problem. Here, as consequence of the nonlinearity given by the convection term, constrains either over small data or small time are necessary in order to obtain the robust control; b) The hierarchical control (second problem), where the main tools will be news Carleman estimates and fixed point arguments for solving the local null controllability associated to the leader control. 
The rest of the paper is organized as follows. In Section 2, we present the general scheme of the robust control problem for the system (1.1). In the first subsection we present the existence and characterization of the robust control for the linearized system (Stokes equation) and in the second subsection the same result for the nonlinear case. In section 3, we solve the robust Stackelberg strategy for the Stokes case. That is, we prove the null controllability for the coupled Stokes system that arises as characterization of the robust control problem. In Section 4, we end the proof of Theorem 1.1 throughout an inverse function theorem of the Lyusternik's kind.

\section{The robust control problem}

As mentioned in the previous section, the main objetive in robust control is to determine the best control function $v \in L^{2}(\mathcal{O} \times(0, T))^{N}$ in the presence of the disturbance $\psi \in L^{2}(Q)^{N}$ which maximally spoils the control. In this section we present some lemmas on the existence, uniqueness and characterisation of a solution to the robust control problem established in Definition 1.1 .

The proof of the existence of a solution $(\bar{\psi}, \bar{v})$ to the robust control problem is based on the following result. The interested reader can see [8] for more details.

Lemma 2.1. Let $\mathcal{J}$ be a functional defined on $X \times Y$, where $X$ and $Y$ are non-empty. closed, unbounded convex sets. If $\mathcal{J}$ satisfies

a) $\forall \psi \in X, v \longmapsto \mathcal{J}(\psi, v)$ is convex lower semicontinuous.

b) $\forall v \in Y, \psi \longmapsto \mathcal{J}(\psi, v)$ is concave upper semicontinuous.

c) $\exists \psi_{0} \in X$ such that $\lim _{\|v\|_{Y} \rightarrow \infty} \mathcal{J}\left(\psi_{0}, v\right)=+\infty$.

d) $\exists v_{0} \in Y$ such that $\lim _{\|\psi\|_{X} \rightarrow \infty} \mathcal{J}\left(\psi, v_{0}\right)=-\infty$.

Then the functional $\mathcal{J}$ has a least one saddle point $(\bar{\psi}, \bar{v})$ and

$$
\mathcal{J}(\bar{\psi}, \bar{v})=\min _{v \in Y} \sup _{\psi \in X} \mathcal{J}(\psi, v)=\max _{\psi \in X} \inf _{v \in Y} \mathcal{J}(\psi, v)
$$

\section{$2.1 \quad$ Linear problem}

In this section we will treat the corresponding robust Stackelberg strategy for the linearized system. That is we will consider the Stokes system

$$
\begin{cases}y_{t}-\Delta y+\nabla p=h 1_{\omega}+v \chi_{\mathcal{O}}+\psi & \text { in } Q, \\ \nabla \cdot y=0 & \text { in } Q \\ y=0 & \text { on } \Sigma, \\ y(\cdot, 0)=y_{0}(\cdot) & \text { in } \Omega .\end{cases}
$$

We have the following result:

Lemma 2.2. Let $h \in L^{2}(\omega \times(0, T))^{N}$ be fixed. There exists $\gamma_{0}>0$ such that for every $\gamma>\gamma_{0}$, there exists a saddle point $(\bar{\psi}, \bar{v})$ and the corresponding solution $y(h, \bar{\psi}, \bar{v})$ of $(2.1)$ such that

$$
J_{r}(\psi, \bar{v} ; h) \leq J_{r}(\bar{\psi}, \bar{v} ; h) \leq J_{r}(\bar{\psi}, v ; h), \forall(\psi, v) \in L^{2}(Q)^{N} \times L^{2}\left(0, T ; L^{2}(\mathcal{O})^{N}\right) .
$$

The proof of Lemma 2.2 follows as in [6] where the authors used Lemma 2.1 with $X=Y=$ $L^{2}(Q)^{N}$ to prove the existence of a saddle point for a slightly different cost functional $\mathcal{J}$. As consequence of this result, the existence of a solution $(\bar{\psi}, \bar{v})$ to our robust control problem is guaranteed. 
Remark 2.1. In lemma 2.2, if the condition on $\gamma$ is not met, we cannot prove the existence of the saddle point. On the other hand, it is known that the existence of a saddle point for the functional $J_{r}$ implies that for any $\psi \in L^{2}(Q)^{N}, v \in L^{2}\left(0, T ; L^{2}(\mathcal{O})^{N}\right)$

$$
\frac{\partial J_{r}}{\partial \psi}(\bar{\psi}, \bar{v}) \cdot \psi=0, \quad \frac{\partial J_{r}}{\partial v}(\bar{\psi}, \bar{v}) \cdot v=0,
$$

where

$$
\frac{\partial J_{r}}{\partial \psi}(\bar{\psi}, \bar{v}) \cdot \psi=\iint_{\mathcal{O}_{d} \times(0, T)}\left(y-y_{d}\right) w_{\psi} d x d t-\gamma^{2} \iint_{\mathcal{O} \times(0, T)} \psi \bar{\psi} d x d t
$$

and

$$
\frac{\partial J_{r}}{\partial v}(\bar{\psi}, \bar{v}) \cdot v=\iint_{\mathcal{O}_{d} \times(0, T)}\left(y-y_{d}\right) w_{v} d x d t+\ell^{2} \iint_{\mathcal{O} \times(0, T)} \chi_{\mathcal{O}} v \bar{v} d x d t,
$$

and $w_{\psi}, w_{v}$ are the Gâteaux derivatives of $y$ solution to (2.1) in the directions $\psi$ and $v$ respectively.

Finally, in order to characterize the robust control problem, we introduce the linear adjoint system to (2.1) with right-hand side related with $J_{r}$, that is, we consider

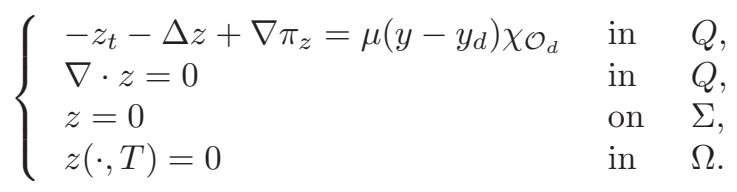

In the following result we characterize the saddle point $(\bar{v}, \bar{\psi})$ in terms of $z$. The interested reader can consult [5] for more details.

Lemma 2.3. Let $h \in L^{2}(\omega \times(0, T))^{N}$ and $y_{0} \in V$ be given. Suppose that $(\bar{\psi}, \bar{v})$ is the solution to the robust control problem stated in Definition 1.1. Then

$$
\bar{\psi}=\frac{1}{\gamma^{2}} z \quad \text { and } \quad \bar{v}=-\frac{1}{\ell^{2}} z \chi_{\mathcal{O}},
$$

where $\gamma$ is sufficiently large and the pair $(y, z)$ solves the following coupled system:

$$
\begin{cases}y_{t}-\Delta y+\nabla \pi_{y}=h 1_{\omega}+\left(-\ell^{-2} \chi_{\mathcal{O}}+\gamma^{-2}\right) z & \text { in } \quad Q, \\ -z_{t}-\Delta z+\nabla \pi_{z}=\mu\left(y-y_{d}\right) \chi_{\mathcal{O}_{d}} & \text { in } Q, \\ \nabla \cdot y=0, \nabla \cdot z=0 & \text { in } \quad Q, \\ y=z=0 & \text { on } \Sigma, \\ y(\cdot, 0)=y_{0}(\cdot), \quad z(\cdot, T)=0 & \text { in } \Omega .\end{cases}
$$

\subsection{Nonlinear problem}

The analysis is similar to the previous one for the linear case. However, it is well known that the theory of the Navier-Stokes equations is complete in two-dimensional spaces, which do not occur in three-dimensional spaces. Roughly speaking, in three dimensions, the existence of a robust control is restricted to cases of either small data or small $T$. Additionally, the nonlinearity will require new assumptions on the parameter $\ell$. Under the constraint of small data, we need to impose the following condition: there exists $\delta>0$ such that, for every $\left(v \chi_{\mathcal{O}}, \psi\right) \in L^{2}(Q)^{N \times N}$ and $y_{0} \in V$

$$
\left\|v \chi_{\mathcal{O}}\right\|_{L^{2}(Q)^{N}}+\|\psi\|_{L^{2}(Q)^{N}} \leq \delta \text { and } \quad\left\|y_{0}\right\|_{V} \leq \delta
$$

holds. 
Lemma 2.4. Let $h \in L^{2}(\omega \times(0, T))^{N}$ be fixed.

i) Case $N=2$. There exist constants $\gamma_{0}>0$ and $\ell_{0}>0$ such that for every $\gamma>\gamma_{0}$ and $\ell>\ell_{0}$, there exists $(\bar{\psi}, \bar{v})$ on $L^{2}(Q)^{N} \times L^{2}\left(0, T ; L^{2}(\mathcal{O})^{N}\right)$ and the associated solution to (1.1) $y=y(h, \bar{v}, \bar{\psi})$ such that

$$
J_{r}(\psi, \bar{v} ; h) \leq J_{r}(\bar{\psi}, \bar{v} ; h) \leq J_{r}(\bar{\psi}, v ; h), \forall(\psi, v) \in L^{2}(Q)^{N} \times L^{2}\left(0, T ; L^{2}(\mathcal{O})^{N}\right) .
$$

That is, $(\bar{\psi}, \bar{v})$ is a saddle point of $J_{r}$.

ii) Case $N=3$. Under the hypothesis of the case $N=2$, and that either $y_{0} \in V$ and $\left(v \chi_{\mathcal{O}}, \psi\right) \in L^{2}(Q)^{N \times N}$ satisfies (2.6), or that $t=T$ is small, then there exists $(\bar{\psi}, \bar{v}) \in$ $L^{2}(Q)^{N} \times L^{2}\left(0, T ; L^{2}(\mathcal{O})^{N}\right)$ a saddle point of $J_{r}$.

Analogously to the linear case, we give the characterization of the robust control problem in the following result.

Lemma 2.5. Let $h \in L^{2}(\omega \times(0, T))$ and $y_{0} \in V$ be given. Then, there exist positive constants $\gamma_{0}, \ell_{0}$ such that if $\gamma>\gamma_{0}, \ell>\ell_{0}$, the solution $(\bar{v}, \bar{\psi})$ to the robust control problem stated in Definition 1.1 exists and is unique. Furthermore, $(\bar{v}, \bar{\psi})$ is characterized by

$$
\bar{\psi}=\frac{1}{\gamma^{2}} z \quad \text { and } \quad \bar{v}=-\frac{1}{\ell^{2}} z \chi_{\mathcal{O}},
$$

where $z$ is the second component of $(y, z)$ solution to the following coupled system:

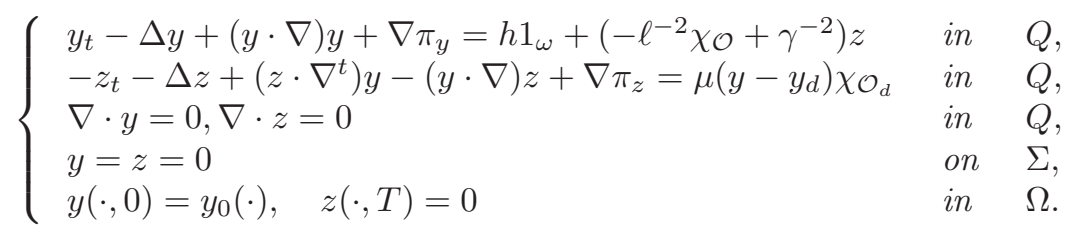

The proof of Lemma 2.4 and Lemma 2.5 can be found in [6].

\section{Controllability}

In the previous sections we saw that the robust control is characterized in such a way that a coupled system needs to be solved. In order to establish a Stackelberg strategy requiring the leader control to drive the equation to zero we need to find $h \in L^{2}(\omega \times(0, T))^{N}$ such that the corresponding $y$ solution to (2.5) (in the linear case) or to (2.8) (in the nonlinear case), satisfies $y(T)=0$. To achieve this objectives, we will obtain first the result in the linear case. To this aim we will prove an observability inequality for the adjoint system to (2.5) by means of Carleman estimates. The nonlinear case will be obtained by a fixed point argument. The next subsection will be devoted to the obtention of the Carleman inequalities.

\subsection{Carleman inequalities}

We first define several weight functions which will be useful in the sequel. Let $\omega_{0}$ be a nonempty open subset of $\mathbb{R}^{N}$ such that $\omega_{0} \Subset \omega \cap \mathcal{O}_{d}$ and $\eta \in C^{2}(\bar{\Omega})$ such that

$$
|\nabla \eta|>0 \text { in } \bar{\Omega} \backslash \omega_{0}, \quad \eta>0 \text { in } \Omega \text { and } \eta \equiv 0 \text { on } \partial \Omega .
$$

The existence of such a function $\eta$ is proved in [11]. Then, for some positive real number $\lambda$, we consider the following weight functions:

$$
\begin{aligned}
& \alpha(x, t)=\frac{e^{12 \lambda\|\eta\|_{\infty}}-e^{\lambda\left(10\|\eta\|_{\infty}+\eta(x)\right)}}{(t(T-t))^{5}}, \quad \xi(x, t)=\frac{e^{\lambda\left(10\|\eta\|_{\infty}+\eta(x)\right)}}{(t(T-t))^{5}}, \\
& \alpha^{*}(t)=\max _{x \in \bar{\Omega}} \alpha(x, t), \quad \xi^{*}(t)=\min _{x \in \bar{\Omega}} \xi(x, t), \\
& \widehat{\alpha}(t)=\min _{x \in \bar{\Omega}} \alpha(x, t), \quad \widehat{\xi}(t)=\max _{x \in \bar{\Omega}} \xi(x, t) .
\end{aligned}
$$


These weight functions have been used by M. Gueye in [15] and S. Guerrero in [14] to obtain Carleman estimates for a Stokes coupled system similar to the presented in our work.

We consider now the non homogeneous adjoint system to (2.5):

$$
\begin{cases}-\varphi_{t}-\Delta \varphi+\nabla \pi_{\varphi}=g_{1}+\mu \theta \chi_{\mathcal{O}_{d}} & \text { in } \quad Q, \\ \theta_{t}-\Delta \theta+\nabla \pi_{\theta}=g_{2}-\ell^{-2} \varphi \chi_{\mathcal{O}}+\gamma^{-2} \varphi & \text { in } \quad Q, \\ \nabla \cdot \varphi=0, \nabla \cdot \theta=0 & \text { in } \quad Q, \\ \varphi=\theta=0 & \text { on } \Sigma, \\ \varphi(\cdot, T)=\varphi_{T}(\cdot), \theta(\cdot, 0)=0 & \text { in } \Omega,\end{cases}
$$

where $g_{1}, g_{2} \in L^{2}(Q)^{N}$ and $\varphi_{T} \in H$.

Our Carleman estimate is given in the following proposition. In what follows, the constants $a_{0}$ and $m_{0}$ are fixed, and satisfy

$$
\frac{5}{4} \leq a_{0}<a_{0}+1<m_{0}<2 a_{0}, \quad m_{0}<2+a_{0} .
$$

Proposition 3.1. Assume that $\omega \cap \mathcal{O}_{d} \neq \emptyset$ and that $\ell$ and $\gamma$ are large enough. Then, there exist a constant $\bar{\lambda}$ such that for any $\lambda \geq \bar{\lambda}$ exist two constants $\bar{s}(\lambda)>0$ and $C=C(\lambda)>0$ depending only on $\Omega$ and $\omega$ such that for any $g_{1}, g_{2} \in L^{2}(Q)^{N}$ and any $\varphi_{T} \in H$, the solution of (3.2) satisfies

$$
\begin{aligned}
& \iint_{Q} e^{-2 s \alpha-2 a_{0} s \alpha^{*}}\left(s \lambda^{2} \xi|\nabla(\nabla \times \theta)|^{2}+s^{3} \lambda^{4} \xi^{3}|\nabla \times \theta|^{2}\right) d x d t \\
& \quad+\iint_{Q} e^{-2 s m_{0} \alpha}\left(s \lambda^{2} \xi|\nabla \varphi|^{2}+s^{3} \lambda^{4} \xi^{3}|\varphi|^{2}+(s \xi)^{-1}|\Delta \varphi|^{2}\right) d x d t \\
& \leq C\left(s^{15} \lambda^{24} \iint_{\omega \times(0, T)} e^{-4 a_{0} s \alpha^{*}+2\left(m_{0}-2\right) s \alpha^{*}}(\hat{\xi})^{15}|\varphi|^{2} d x d t\right. \\
& \left.\quad+s^{5} \lambda^{6} \iint_{Q} e^{-2 s \hat{\alpha}-2 a_{0} s \alpha^{*}}(\hat{\xi})^{5}\left|g_{1}\right|^{2} d x d t+\iint_{Q} e^{-2 a_{0} s \alpha^{*}}\left|g_{2}\right|^{2} d x d t\right),
\end{aligned}
$$

for any $s \geq \bar{s}$.

Before giving the proof of Proposition 3.1, we recall some technical results. We first present a Carleman inequality proved in $[9]$ for a general heat equation with Fourier boundary conditions. Let us introduce the system

$$
\left\{\begin{array}{lll}
-u_{t}-\Delta u=f_{1}+\nabla \cdot f_{2} & \text { in } & Q, \\
\left(\nabla u+f_{2}\right) \cdot n=f_{3} & \text { on } & \Sigma, \\
u(\cdot, T)=u_{T}(\cdot) & \text { in } & \Omega,
\end{array}\right.
$$

where $f_{1} \in L^{2}(Q), f_{2} \in L^{2}(Q)^{N}$ and $f_{3} \in L^{2}(\Sigma)$. We have:

Lemma 3.1. Under the previous assumptions on $f_{1}, f_{2}$ and $f_{3}$, there exist positive constants $\bar{\lambda}, \sigma_{1}, \sigma_{2}$ and $C$, only depending on $\Omega$ and $\omega$, such that, for any $\lambda \geq \bar{\lambda}$, any $s \geq \bar{s}=\sigma_{1}\left(e^{\sigma_{2} \lambda} T+\right.$ $T^{2}$ ) and any $u_{T} \in L^{2}(\Omega)$, the weak solution to (3.5) satisfies

$$
\begin{gathered}
\iint_{Q} e^{-2 s \alpha}\left[s^{3} \lambda^{4} \xi^{3}|u|^{2}+s \lambda^{2} \xi|\nabla u|^{2}\right] d x d t \leq C\left(\iint_{Q} e^{-2 s \alpha}\left(\left|f_{1}\right|^{2}+s^{2} \lambda^{2} \xi^{2}\left|f_{2}\right|^{2}\right) d x d t\right. \\
\left.+s \lambda \iint_{\Sigma} e^{-2 s \alpha} \xi\left|f_{3}\right|^{2} d \sigma d t+s^{3} \lambda^{4} \iint_{\omega_{0} \times(0, T)} e^{-2 s \alpha} \xi^{3}|u|^{2} d x d t\right) .
\end{gathered}
$$


The second result holds for the solutions of a Stokes system with Dirichlet boundary conditions. The interested reader can see [10] for more details.

Lemma 3.2. Let $u_{0} \in V$ and $f_{4} \in L^{2}(Q)^{N}$. Then, there exists a constant $C(\Omega, \omega, T)>0$ such that the solution $(u, p) \in L^{2}\left(0, T ; H^{2}(\Omega)^{N} \cap V\right) \cap L^{\infty}(0, T ; V) \times L^{2}\left(0, T ; H^{1}(\Omega)\right)$, with $\int_{\omega_{0}} p(x, t) d x=0$, of

$$
\begin{cases}u_{t}-\Delta u+\nabla p=f_{4} & \text { in } \quad Q \\ \nabla \cdot u=0 & \text { in } \quad Q \\ u=0 & \text { on } \quad \Sigma \\ u(\cdot, 0)=u_{0}(\cdot) & \text { in } \quad \Omega\end{cases}
$$

satisfies

$$
\begin{aligned}
& \iint_{Q} e^{-2 s \alpha}\left(s \lambda^{2} \xi|\nabla u|^{2}+s^{3} \lambda^{4} \xi^{3}|u|^{2}\right) d x d t \\
& \leq C\left(s^{16} \lambda^{40} \iint_{\omega \times(0, T)} e^{-8 s \hat{\alpha}+6 s \alpha^{*}}(\hat{\xi})^{16}|u|^{2} d x d t+s^{15 / 2} \lambda^{20} \iint_{Q} e^{-4 s \hat{\alpha}+2 s \alpha^{*}}(\hat{\xi})^{15 / 2}\left|f_{4}\right|^{2} d x d t\right),
\end{aligned}
$$

for any $\lambda \geq C$ and $s \geq C\left(T^{5}+T^{10}\right)$.

Remark 3.1. In [9] and [10] slightly different weight functions are used to prove the above results. However, the inequality remains valid since the key point of the proof is that $\alpha$ goes to 0 when $t$ tends to 0 and $T$.

The next result concerns the regularity of the solutions to the Stokes system, see [21] and [14] for more details.

Lemma 3.3. Let $a \in \mathbb{R}$ and $B \in \mathbb{R}^{N}$ be constant and let $f_{5} \in L^{2}(0, T ; V)$. Then, there exists a unique solution

$$
u \in L^{2}\left(0, T ; H^{3}(\Omega)^{N} \cap V\right) \cap H^{1}(0, T ; V)
$$

for the Stokes system

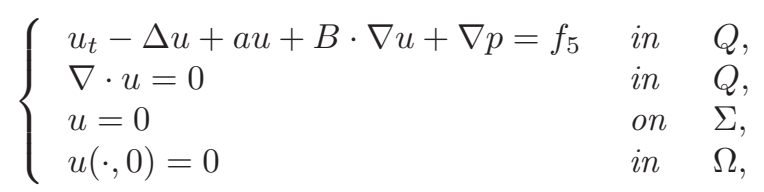

for some $p \in L^{2}\left(0, T ; H^{2}(\Omega)\right)$, and there exists a constant $C>0$ such that

$$
\|u\|_{L^{2}\left(0, T ; H^{3}(\Omega)^{N}\right)}+\|u\|_{H^{1}\left(0, T ; L^{2}(\Omega)^{N}\right)} \leq C\left\|f_{5}\right\|_{L^{2}\left(0, T ; H^{1}(\Omega)^{N}\right)} .
$$

Moreover, if we assume that $a \equiv B \equiv 0$ and $f_{5} \in L^{2}(Q)^{N}, u$ is actually, together a pressure $p$, the strong solution of $(3.8)$, i.e., $(u, p) \in L^{2}\left(0, T ; H^{2}(\Omega)^{N}\right) \cap L^{\infty}(0, T ; V) \cap H^{1}(0, T ; H) \times$ $L^{2}\left(0, T ; H^{1}(\Omega)\right)$. Furthermore, there exists a constant $C>0$ such that

$$
\|u\|_{L^{2}\left(0, T ; H^{2}(\Omega)^{N}\right)}+\|u\|_{L^{\infty}(0, T ; V)}+\|u\|_{H^{1}\left(0, T ; L^{2}(\Omega)^{N}\right)} \leq C\left\|f_{5}\right\|_{L^{2}(Q)^{N}} .
$$

Now, we give the proof of Proposition 3.1.

\subsection{Proof of Proposition 3.1}

\section{Carleman estimate for $\theta$}


Let define $\theta^{*}:=\rho^{*} \theta, \quad \pi^{*}:=\rho^{*} \pi$, where $\rho^{*}=\rho^{*}(t)=e^{-a_{0} s \alpha^{*}}$ and $a_{0}$ fixed satisfying (3.3). From $(3.2),\left(\theta^{*}, \pi^{*}\right)$ is the solution of the following system

$$
\begin{cases}\theta_{t}^{*}-\Delta \theta^{*}+\nabla \pi^{*}=\rho^{*} g_{2}+\rho^{*}\left(-\ell^{-2} \varphi \chi_{\mathcal{O}}+\gamma^{-2} \varphi\right)+\rho_{t}^{*} \theta & \text { in } Q, \\ \nabla \cdot \theta^{*}=0 & \text { in } Q, \\ \theta^{*}=0 & \text { on } \Sigma, \\ \theta^{*}(\cdot, 0)=0 & \text { in } \Omega .\end{cases}
$$

Now, we decompose $\left(\theta^{*}, \pi^{*}\right)$ as follows:

$$
\left(\theta^{*}, \pi^{*}\right)=(\hat{\theta}, \hat{\pi})+(\tilde{\theta}, \tilde{\pi})
$$

where $(\hat{\theta}, \hat{\pi})$ and $(\tilde{\theta}, \tilde{\pi})$ solve respectively

$$
\begin{cases}\tilde{\theta}_{t}-\Delta \tilde{\theta}+\nabla \tilde{\pi}=\rho^{*} g_{2}+\rho^{*}\left(-\ell^{-2} \varphi \chi_{\mathcal{O}}+\gamma^{-2} \varphi\right) & \text { in } \quad Q, \\ \nabla \cdot \tilde{\theta}=0 & \text { in } \quad Q, \\ \tilde{\theta}=0 & \text { on } \Sigma, \\ \tilde{\theta}(\cdot, 0)=0 & \text { in } \Omega,\end{cases}
$$

and

$$
\left\{\begin{array}{lll}
\hat{\theta}_{t}-\Delta \hat{\theta}+\nabla \hat{\pi}=\rho_{t}^{*} \theta & \text { in } \quad Q, \\
\nabla \cdot \hat{\theta}=0 & \text { in } \quad Q, \\
\hat{\theta}=0 & \text { on } \quad \Sigma, \\
\hat{\theta}(\cdot, 0)=0 & \text { in } \quad \Omega .
\end{array}\right.
$$

For system (3.12) we will use Lemma 3.3 and the regularity result estimate (3.10), meanwhile for the system (3.13) we will use the ideas of both works [14] and [15].

We apply the operator $\nabla \times \cdot$ to the Stokes system satisfied by $\hat{\theta}$. Then, we have

$$
(\nabla \times \hat{\theta})_{t}-\Delta(\nabla \times \hat{\theta})=\rho_{t}^{*}(\nabla \times \theta) \text { in } Q .
$$

Using Lemma [9] with $f_{1}=\rho_{t}^{*}(\nabla \times \theta)$, there exists a constant $C=C\left(\Omega, \omega_{0}\right)>0$ such that

$$
\begin{aligned}
& \iint_{Q} e^{-2 s \alpha}\left(s \lambda^{2} \xi|\nabla(\nabla \times \hat{\theta})|^{2}+s^{3} \lambda^{4} \xi^{3}|\nabla \times \hat{\theta}|^{2}\right) d x d t \\
& \leq C\left(\iint_{Q} e^{-2 s \alpha}\left|\rho_{t}^{*}\right|^{2}|\nabla \times \theta|^{2} d x d t\right. \\
& \left.\quad+s \lambda \iint_{\Sigma} e^{-2 s \alpha} \xi\left|\frac{\partial(\nabla \times \hat{\theta})}{\partial n}\right|^{2} d \sigma d t+s^{3} \lambda^{4} \iint_{\omega_{0} \times(0, T)} e^{-2 s \alpha} \xi^{3}|\nabla \times \hat{\theta}|^{2} d x d t\right),
\end{aligned}
$$

for any $\lambda \geq C$ and $s \geq C\left(T^{10}+T^{9}\right)$.

Now, using the inequality $(a-b)^{2} \geq \frac{a^{2}}{2}-b^{2}$, for every $a, b \in \mathbb{R}$ with $a=\theta^{*}$ and $b=\tilde{\theta}$, we get (recall that $\left.\hat{\theta}=\theta^{*}-\tilde{\theta}\right)$ :

$$
\begin{aligned}
& \frac{1}{2} \iint_{Q} e^{-2 s \alpha-2 a_{0} s \alpha^{*}}\left(s \lambda^{2} \xi|\nabla(\nabla \times \theta)|^{2}+s^{3} \lambda^{4} \xi^{3}|\nabla \times \theta|^{2}\right) d x d t \\
& \quad-\iint_{Q} e^{-2 s \alpha}\left(s \lambda^{2} \xi|\nabla(\nabla \times \tilde{\theta})|^{2}+s^{3} \lambda^{4} \xi^{3}|\nabla \times \tilde{\theta}|^{2}\right) d x d t \\
& \leq \iint_{Q} e^{-2 s \alpha}\left(s \lambda^{2} \xi|\nabla(\nabla \times \hat{\theta})|^{2}+s^{3} \lambda^{4} \xi^{3}|\nabla \times \hat{\theta}|^{2}\right) d x d t .
\end{aligned}
$$


The fact that $s^{3} \lambda^{4} e^{-2 s \alpha} \xi^{3}$ and $s \lambda^{2} e^{-2 s \alpha} \xi$ are upper bounded allow us to estimate the terms associated to $|\nabla(\nabla \times \tilde{\theta})|^{2}$ and $|\nabla \times \tilde{\theta}|^{2}$ through (3.10). More precisely, we have:

$$
\begin{aligned}
& s^{3} \lambda^{4} \iint_{Q} e^{-2 s \alpha} \xi^{3}|\nabla \times \tilde{\theta}|^{2} d x d t+s \lambda^{2} \iint_{Q} e^{-2 s \alpha} \xi|\nabla(\nabla \times \tilde{\theta})|^{2} d x d t \\
& \leq C_{s, \lambda}\|\tilde{\theta}\|_{L^{2}\left(0, T ; H^{1}(\Omega)^{N}\right) \cap L^{2}\left(0, T ; H^{2}(\Omega)^{N}\right)}^{2} \\
& \leq C_{s, \lambda}\left\|\rho^{*} g_{2}\right\|_{L^{2}(Q)^{N}}^{2}+C_{s, \lambda}\left\|\rho^{*}\left(-\ell^{-2} \varphi \chi_{\mathcal{O}}+\gamma^{-2} \varphi\right)\right\|_{L^{2}(Q)^{N}}^{2},
\end{aligned}
$$

where $C_{s, \lambda}$ is a positive constant depending on $s$ and $\lambda$. that

On the other hand, taking into account that $\left|\rho_{t}^{*}\right| \leq C s T \rho^{*}\left(\xi^{*}\right)^{6 / 5}$ for every $s \geq C$, it follows

$$
\iint_{Q} e^{-2 s \alpha}\left|\rho_{t}^{*}\right|^{2}|\nabla \times \theta|^{2} d x d t \leq C s^{2} T^{2} \iint_{Q} e^{-2 s \alpha-2 a_{0} s \alpha^{*}}\left(\xi^{*}\right)^{12 / 5}|\nabla \times \theta|^{2} d x d t,
$$

which can be absorbed by the first term in the right-hand side of (3.15), for every $\lambda \geq 1, s \geq C$. From the identity $\theta^{*}=\hat{\theta}+\tilde{\theta}$ (recall (3.11)) and (3.16), it is easy to estimate the local term that appear in the right-hand side of (3.14) by:

$$
\begin{aligned}
& s^{3} \lambda^{4} \iint_{\omega_{0} \times(0, T)} e^{-2 s \alpha} \xi^{3}|\nabla \times \hat{\theta}|^{2} d x d t \\
& \leq C\left(s^{3} \lambda^{4} \iint_{\omega_{0} \times(0, T)} e^{-2 s \alpha} \xi^{3}\left(\left|\nabla \times \theta^{*}\right|^{2}+|\nabla \times \tilde{\theta}|^{2}\right) d x d t\right) \\
& \leq C s^{3} \lambda^{4} \iint_{\omega_{0} \times(0, T)} e^{-2 s \alpha} \xi^{3}\left|\nabla \times \theta^{*}\right|^{2} d x d t+C_{s, \lambda}\left\|\rho^{*} g_{2}\right\|_{L^{2}(Q)^{N}}^{2}+C_{s, \lambda}\left\|\rho^{*}\left(-\ell^{-2} \varphi \chi_{\mathcal{O}}+\gamma^{-2} \varphi\right)\right\|_{L^{2}(Q)^{N}}^{2} .
\end{aligned}
$$

Putting together (3.14), (3.15) and (3.17), we have for the moment

$$
\begin{aligned}
& \iint_{Q} e^{-2 s \alpha-2 a_{0} s \alpha^{*}}\left(s \lambda^{2} \xi|\nabla(\nabla \times \theta)|^{2}+s^{3} \lambda^{4} \xi^{3}|\nabla \times \theta|^{2}\right) d x d t \\
& \leq C\left(s^{3} \lambda^{4} \iint_{\omega_{0} \times(0, T)} e^{-2 s \alpha-2 a_{0} s \alpha^{*}} \xi^{3}|\nabla \times \theta|^{2} d x d t+s \lambda \iint_{\Sigma} e^{-2 s \alpha} \xi\left|\frac{\partial(\nabla \times \hat{\theta})}{\partial n}\right|^{2} d \sigma d t\right) \\
& \quad+C_{s, \lambda}\left\|\rho^{*} g_{2}\right\|_{L^{2}(Q)^{N}}^{2}+C_{s, \lambda}\left\|\rho^{*}\left(-\ell^{-2} \varphi \chi_{\mathcal{O}}+\gamma^{-2} \varphi\right)\right\|_{L^{2}(Q)^{N}}^{2},
\end{aligned}
$$

for every $s \geq C$ and $\lambda \geq C$.

The last step will be to estimate the boundary term

$$
s \lambda \iint_{\Sigma} e^{-2 s \alpha} \xi\left|\frac{\partial(\nabla \times \hat{\theta})}{\partial n}\right|^{2} d \sigma d t .
$$

To this end we follow the arguments of [15]. For brevity we omitted the calculus but refer to [15].

Therefore, there exist $C_{s, \lambda}>0$ and $C>0$ such that

$$
\begin{aligned}
s \lambda \iint_{\Sigma} e^{-2 s \alpha} \xi\left|\frac{\partial(\nabla \times \hat{\theta})}{\partial n}\right|^{2} d \sigma d t & \leq C\left\|\rho^{*} g_{2}\right\|_{L^{2}(Q)^{N}}^{2}+C_{s, \lambda}\left\|\rho^{*}\left(-\ell^{-2} \varphi \chi_{\mathcal{O}}+\gamma^{-2} \varphi\right)\right\|_{L^{2}(Q)^{N}}^{2} \\
& +\varepsilon\left(\iint_{Q} e^{-2 s \alpha-2 a_{0} s \alpha^{*}}\left(s \lambda^{2} \xi|\nabla(\nabla \times \theta)|^{2}+s^{3} \lambda^{4} \xi^{3}|\nabla \times \theta|^{2}\right) d x d t\right),
\end{aligned}
$$


for every $\varepsilon>0$.

From the previous inequality and (3.18) we conclude the following Carleman estimate for $\theta$ :

$$
\begin{aligned}
& \iint_{Q} e^{-2 s \alpha-2 a_{0} s \alpha^{*}}\left(s \lambda^{2} \xi|\nabla(\nabla \times \theta)|^{2}+s^{3} \lambda^{4} \xi^{3}|\nabla \times \theta|^{2}\right) d x d t \leq C\left\|\rho^{*} g_{2}\right\|_{L^{2}(Q)^{N}}^{2} \\
& +C_{s, \lambda}\left\|\rho^{*}\left(-\ell^{-2} \varphi \chi_{\mathcal{O}}+\gamma^{-2} \varphi\right)\right\|_{L^{2}(Q)^{N}}^{2}+C s^{3} \lambda^{4} \iint_{\omega_{0} \times(0, T)} e^{-2 s \alpha-2 a_{0} s \alpha^{*}} \xi^{3}|\nabla \times \theta|^{2} d x d t,
\end{aligned}
$$

for every $s \geq C\left(T^{5}+T^{10}\right)$ and $\lambda \geq C$.

\section{Carleman estimate for $\varphi$}

First, assuming that $\theta$ is given, we look at $\varphi$ as the solution of

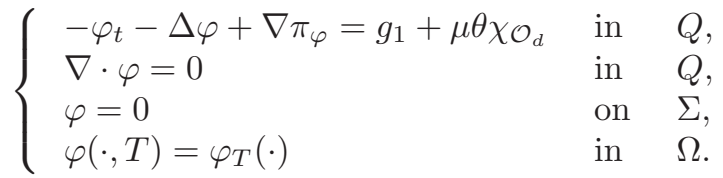

Now, we choose $\pi_{\varphi}$ such that $\int_{\omega_{0}} \pi_{\varphi} d x=0$ and we apply Lemma 3.2 with $f_{4}=g_{1}+\mu \theta \chi_{\mathcal{O}_{d}}$ and use the weight function $m_{0} \alpha$ (instead of $\alpha$ ), where $a_{0}+1<m_{0} \leq 2 a_{0}$ and $m_{0} \leq 2+a_{0}$. We obtain

$$
\begin{gathered}
\iint_{Q} e^{-2 m_{0} s \alpha}\left[s^{-1} \xi^{-1}|\Delta \varphi|^{2}+s \lambda^{2} \xi|\nabla \varphi|^{2}+s^{3} \lambda^{4} \xi^{3}|\varphi|^{2}\right] d x d t \\
\leq C\left(s^{16} \lambda^{40} \iint_{\omega_{0} \times(0, T)} e^{-8 m_{0} s \hat{\alpha}+6 m_{0} s \alpha^{*}}(\hat{\xi})^{16}|\varphi|^{2} d x d t\right. \\
+s^{15 / 2} \lambda^{20} \iint_{\mathcal{O}_{d} \times(0, T)} e^{-4 m_{0} s \hat{\alpha}+2 m_{0} s \alpha^{*}}(\hat{\xi})^{15 / 2}|\theta|^{2} d x d t \\
\left.+s^{15 / 2} \lambda^{20} \iint_{Q} e^{-4 m_{0} s \hat{\alpha}+2 m_{0} s \alpha^{*}}(\hat{\xi})^{15 / 2}\left|g_{1}\right|^{2} d x d t\right),
\end{gathered}
$$

for any $\lambda \geq C$ and $s \geq C\left(T^{5}+T^{10}\right)$.

Taking into account that $\|\theta\|_{L^{2}(\Omega)^{N}} \leq C\|\nabla \times \theta\|_{L^{2}(\Omega)^{2 N-3}}$ and the inequality (6.1) with $\varepsilon=\frac{m_{0}-a_{0}-1}{m_{0}+a_{0}+1}, M_{1}=-\frac{15}{4\left(m_{0}+a_{0}+1\right)}$ and $M_{2}=-\frac{10}{\left(m_{0}+a_{0}+1\right)}$, the second term in the right-hand side of (3.20) can be estimated by

$$
\iint_{Q} e^{-2 s \alpha^{*}-2 a_{0} s \alpha^{*}}|\nabla \times \theta|^{2} d x d t
$$

and therefore it can be absorbed by the left-hand side of (3.19). 
From (3.19) and (3.20) we have

$$
\begin{aligned}
& \iint_{Q} e^{-2 s \alpha-2 a_{0} s \alpha^{*}}\left(s \lambda^{2} \xi|\nabla(\nabla \times \theta)|^{2} d x d t+s^{3} \lambda^{4} \xi^{3}|\nabla \times \theta|^{2}\right) d x d t \\
& +\iint_{Q} e^{-2 m_{0} s \alpha}\left[s^{-1} \xi^{-1}|\Delta \varphi|^{2}+s \lambda^{2} \xi|\nabla \varphi|^{2}+s^{3} \lambda^{4} \xi^{3}|\varphi|^{2}\right] d x d t \\
& \leq C s^{16} \lambda^{40} \iint_{\omega_{0} \times(0, T)} e^{-8 m_{0} s \hat{\alpha}+6 m_{0} s \alpha^{*}}(\hat{\xi})^{16}|\varphi|^{2} d x d t+C_{s, \lambda}\left\|\rho^{*}\left(-\ell^{-2} \varphi \chi_{\mathcal{O}}+\gamma^{-2} \varphi\right)\right\|_{L^{2}(Q)^{N}}^{2} \\
& \quad+C s^{15 / 2} \lambda^{20} \iint_{Q} e^{-4 m_{0} s \hat{\alpha}+2 m_{0} s \alpha^{*}}(\hat{\xi})^{15 / 2}\left|g_{1}\right|^{2} d x d t+C\left\|\rho^{*} g_{2}\right\|_{L^{2}(Q)^{N}}^{2} \\
& \quad+C s^{3} \lambda^{4} \iint_{\omega_{0} \times(0, T)} e^{-2 s \alpha-2 a_{0} s \alpha^{*}} \xi^{3}|\nabla \times \theta|^{2} d x d t,
\end{aligned}
$$

for any $\lambda \geq C, s \geq C\left(T^{5}+T^{10}\right)$ and $C_{s, \lambda}$ depending on $s, \lambda$.

Choosing $\ell$ and $\gamma$ large enough $\left(\ell, \gamma \approx s^{4} \lambda^{5} e^{\lambda\|\eta\|_{\infty}}\right)$, we can absorb the second term in the right-hand side of (3.21) by the left-hand side.

Let us estimate the local term concerning $\nabla \times \theta$ in terms of $\varphi$. To do this, we use the first equation of (3.2) since $\omega \cap \mathcal{O}_{d} \neq \emptyset$ and $\omega_{0} \subset \mathcal{O}_{d}$. We have

$$
-(\nabla \times \varphi)_{t}-\Delta(\nabla \times \varphi)=\nabla \times g_{1}+\mu(\nabla \times \theta), \quad \text { in } \omega_{0} \times(0, T) .
$$

Then,

$$
\begin{aligned}
I & :=s^{3} \lambda^{4} \iint_{\omega_{0} \times(0, T)} e^{-2 s \alpha-2 a_{0} s \alpha^{*}} \xi^{3}|\nabla \times \theta|^{2} d x d t \\
& =s^{3} \lambda^{4} \iint_{\omega_{0} \times(0, T)} e^{-2 s \alpha-2 a_{0} s \alpha^{*}} \xi^{3}(\nabla \times \theta)\left(-(\nabla \times \varphi)_{t}-\Delta(\nabla \times \varphi)-\left(\nabla \times g_{1}\right)\right) d x d t .
\end{aligned}
$$

We introduce an open set $\omega_{1} \Subset \omega$ such that $\omega_{0} \Subset \omega_{1}$ and a positive function $\zeta \in C_{c}^{2}\left(\omega_{1}\right)$ such that $\zeta \equiv 1$ in $\omega_{0}$. Then, after several integration by parts in time and space we have:

$$
\begin{gathered}
I=s^{3} \lambda^{4} \iint_{\omega_{0} \times(0, T)} e^{-2 s \alpha-2 a_{0} s \alpha^{*}} \xi^{3}(\nabla \times \theta)\left(-(\nabla \times \varphi)_{t}-\Delta(\nabla \times \varphi)-\left(\nabla \times g_{1}\right)\right) d x d t \\
\leq s^{3} \lambda^{4} \iint_{\omega_{1} \times(0, T)} \zeta \partial_{t}\left(e^{-2 s \alpha-2 a_{0} s \alpha^{*}} \xi^{3}\right)(\nabla \times \theta)(\nabla \times \varphi) d x d t \\
+s^{3} \lambda^{4} \iint_{\omega_{1} \times(0, T)} e^{-2 s \alpha-2 a_{0} s \alpha^{*}} \xi^{3}\left((\nabla \times \theta)_{t}-\Delta(\nabla \times \theta)\right)(\nabla \times \varphi) d x d t \\
-s^{3} \lambda^{4} \iint_{\omega_{1} \times(0, T)} \Delta\left(\zeta e^{-2 s \alpha-2 a_{0} s \alpha^{*}} \xi^{3}\right)(\nabla \times \theta)(\nabla \times \varphi) d x d t \\
-2 s^{3} \lambda^{4} \iint_{\omega_{1} \times(0, T)} \nabla\left(\zeta e^{-2 s \alpha-2 a_{0} s \alpha^{*}} \xi^{3}\right)(\nabla(\nabla \times \theta))(\nabla \times \varphi) d x d t \\
-s^{3} \lambda^{4} \int_{\omega_{1} \times(0, T)} \zeta e^{-2 s \alpha-2 a_{0} s \alpha^{*}} \xi^{3}(\nabla \times \theta)\left(\nabla \times g_{1}\right) d x d t .
\end{gathered}
$$


From the second equation in (3.2), we have that

$$
\begin{aligned}
I \leq s^{3} \lambda^{4} \iint_{\omega_{1} \times(0, T)} \zeta \partial_{t}\left(e^{-2 s \alpha-2 a_{0} s \alpha^{*}} \xi^{3}\right)(\nabla \times \theta)(\nabla \times \varphi) d x d t \\
+s^{3} \lambda^{4} \gamma^{-2} \iint_{\omega_{1} \times(0, T)} e^{-2 s \alpha-2 a_{0} s \alpha^{*}} \xi^{3}|\nabla \times \varphi|^{2} d x d t \\
\quad-s^{3} \lambda^{4} \iint_{\omega_{1} \times(0, T)} \Delta\left(\zeta e^{-2 s \alpha-2 a_{0} s \alpha^{*}} \xi^{3}\right)(\nabla \times \theta)(\nabla \times \varphi) d x d t \\
\quad-2 s^{3} \lambda^{4} \iint_{\omega_{1} \times(0, T)} \nabla\left(\zeta e^{-2 s \alpha-2 a_{0} s \alpha^{*}} \xi^{3}\right)(\nabla(\nabla \times \theta))(\nabla \times \varphi) d x d t \\
\quad-s^{3} \lambda^{4} \iint_{\omega_{1} \times(0, T)} \zeta e^{-2 s \alpha-2 a_{0} s \alpha^{*}} \xi^{3}(\nabla \times \theta)\left(\nabla \times g_{1}\right) d x d t \\
\quad-s^{3} \lambda^{4} \iint_{\omega_{1} \times(0, T)} \zeta e^{-2 s \alpha-2 a_{0} s \alpha^{*}} \xi^{3}(\nabla \times \varphi)\left(\nabla \times g_{2}\right) d x d t .
\end{aligned}
$$

Using the estimate

$$
\left|\partial_{t}\left(e^{-2 s \alpha-2 a_{0} s \alpha^{*}} \xi^{3}\right)\right| \leq C T s e^{-2 s \alpha-2 a_{0} s \alpha^{*}}(\xi)^{4+1 / 5}, \quad \text { for every } s \geq C
$$

and Young's inequality, we can deduce the following inequalities:

$$
\begin{aligned}
I_{1} & :=s^{3} \lambda^{4} \iint_{\omega_{1} \times(0, T)} \zeta \partial_{t}\left(e^{-2 s \alpha-2 a_{0} s \alpha^{*}} \xi^{3}\right)(\nabla \times \theta)(\nabla \times \varphi) d x d t \\
\leq & C T s^{4} \lambda^{4} \iint_{\omega_{1} \times(0, T)} \zeta e^{-2 s \alpha-2 a_{0} s \alpha^{*}} \xi^{4+1 / 5}|\nabla \times \theta||\nabla \times \varphi| d x d t \\
\leq & \varepsilon s^{3} \iint_{\omega_{1} \times(0, T)} e^{-2 s \alpha-2 a_{0} s \alpha^{*}} \xi^{3}|\nabla \times \theta|^{2} d x d t \\
& +C(\varepsilon) s^{5} \lambda^{8} \iint_{\omega_{1} \times(0, T)} e^{-2 s \alpha-2 a_{0} s \alpha^{*}}(\xi)^{5+2 / 5}|\nabla \times \varphi|^{2} d x d t
\end{aligned}
$$

for every $s \geq C$ and every $\varepsilon>0$.

Now, using the estimate

$$
\left|\Delta\left(\zeta e^{-2 s \alpha-2 a_{0} s \alpha^{*}} \xi^{3}\right)\right| \leq C s^{2} \lambda^{2} e^{-2 s \alpha-2 a_{0} s \alpha^{*}} \xi^{5}, \quad \text { for every } s \geq C
$$

and again the Young's inequality for the third term in the right-hand side of (3.22), we obtain

$$
\begin{aligned}
I_{3} & :=-s^{3} \lambda^{4} \iint_{\omega_{1} \times(0, T)} \Delta\left(\zeta e^{-2 s \alpha-2 a_{0} s \alpha^{*}} \xi^{3}\right)(\nabla \times \theta)(\nabla \times \varphi) d x d t \\
& \leq C s^{5} \lambda^{6} \iint_{\omega_{1} \times(0, T)} e^{-2 s \alpha-2 a_{0} s \alpha^{*}} \xi^{5}|\nabla \times \theta||\nabla \times \varphi| d x d t \\
& \leq \varepsilon s^{3} \iint_{\omega_{1} \times(0, T)} e^{-2 s \alpha-2 a_{0} s \alpha^{*}} \xi^{3}|\nabla \times \theta|^{2} d x d t+C(\varepsilon) s^{7} \lambda^{12} \iint_{\omega_{1} \times(0, T)} e^{-2 s \alpha-2 a_{0} s \alpha^{*}} \xi^{7}|\nabla \times \varphi|^{2} d x d t
\end{aligned}
$$

for every $s \geq C$ and every $\varepsilon>0$. 
Analogously, we can estimate the fourth term in the right-hand side of (3.22) by

$$
\begin{aligned}
I_{4} & :=-2 s^{3} \lambda^{4} \iint_{\omega_{1} \times(0, T)} \nabla\left(\zeta e^{-2 s \alpha-2 a_{0} s \alpha^{*}} \xi^{3}\right)(\nabla(\nabla \times \theta))(\nabla \times \varphi) d x d t \\
& \leq \varepsilon s \lambda^{2} \iint_{\omega_{1} \times(0, T)} e^{-2 s \alpha-2 a_{0} s \alpha^{*}} \xi|\nabla(\nabla \times \theta)|^{2} d x d t+C(\varepsilon) s^{7} \lambda^{8} \iint_{\omega_{1} \times(0, T)} e^{-2 s \alpha-2 a_{0} s \alpha^{*}} \xi^{7}|\nabla \times \varphi|^{2} d x d t,
\end{aligned}
$$

for every $s \geq C$ and every $\varepsilon>0$.

Additionally, through another integration by part and Young's inequality we can obtain

$$
\begin{aligned}
& I_{5} \quad:=-s^{3} \lambda^{4} \iint_{\omega_{1} \times(0, T)} \zeta e^{-2 s \alpha-2 a_{0} s \alpha^{*}} \xi^{3}(\nabla \times \theta)\left(\nabla \times g_{1}\right) d x d t \\
& \leq \varepsilon\left(s \lambda^{2} \iint_{\omega_{1} \times(0, T)} e^{-2 s \alpha-2 a_{0} s \alpha^{*}} \xi|\nabla(\nabla \times \theta)|^{2} d x d t+s^{3} \lambda^{4} \iint_{\omega_{1} \times(0, T)} e^{-2 s \alpha-2 a_{0} s \alpha^{*}} \xi^{3}|\nabla \times \theta|^{2} d x d t\right) \\
& \quad+C(\varepsilon) s^{5} \lambda^{6} \iint_{Q} e^{-2 s \alpha-2 a_{0} s \alpha^{*}} \xi^{5}\left|g_{1}\right|^{2} d x d t,
\end{aligned}
$$

for every $s \geq C$ and every $\varepsilon>0$.

$$
\begin{gathered}
I_{6} \quad:=-s^{3} \lambda^{4} \iint_{\omega_{1} \times(0, T)} \zeta e^{-2 s \alpha-2 a_{0} s \alpha^{*}} \xi^{3}(\nabla \times \varphi)\left(\nabla \times g_{2}\right) d x d t \\
\leq C\left(\left\|\rho^{*} g_{2}\right\|_{L^{2}(Q)^{N}}+s^{7} \lambda^{12} \iint_{\omega_{1} \times(0, T)} e^{-2 s \alpha-2 a_{0} s \alpha^{*}} \xi^{7}|\nabla \varphi|^{2} d x d t\right. \\
\left.\quad+s^{6} \lambda^{8} \iint_{\omega_{1} \times(0, T)} e^{-4 s \alpha-2 a_{0} s \alpha^{*}} \xi^{6}|\nabla \times(\nabla \times \varphi)|^{2} d x d t\right) .
\end{gathered}
$$

We use Lemma 6.2 in the Appendix in order to obtain an appropriate upper bound for the last term in the right-hand side on the previous inequality

$$
s^{7} \lambda^{12} \iint_{\omega_{1} \times(0, T)} e^{-2 s \alpha-2 a_{0} s \alpha^{*}} \xi^{7}|\nabla \varphi|^{2} d x d t \quad \text { and } \quad \varepsilon s^{-1} \iint_{Q} e^{-2 m_{0} s \alpha} \xi^{-1}|\Delta \varphi|^{2} d x d t,
$$

for every $\varepsilon>0$.

Putting together (3.21) and the previous estimates, we have

$$
\begin{aligned}
& \iint_{Q} e^{-2 s \alpha-2 a_{0} s \alpha^{*}}\left(s \lambda^{2} \xi|\nabla(\nabla \times \theta)|^{2} d x d t+s^{3} \lambda^{4} \xi^{3}|\nabla \times \theta|^{2}\right) d x d t \\
& +\iint_{Q} e^{-2 m_{0} s \alpha}\left[s^{-1} \xi^{-1}|\Delta \varphi|^{2}+s \lambda^{2} \xi|\nabla \varphi|^{2}+s^{3} \lambda^{4} \xi^{3}|\varphi|^{2}\right] d x d t \\
& \leq C s^{16} \lambda^{40} \iint_{\omega_{0} \times(0, T)} e^{-8 m_{0} s \hat{\alpha}+6 m_{0} s \alpha^{*}}(\hat{\xi})^{16}|\varphi|^{2} d x d t+C \iint_{Q} e^{-2 a_{0} s \alpha^{*}}\left|g_{2}\right|^{2} d x d t \\
& +C s^{15 / 2} \lambda^{20} \iint_{Q} e^{-4 m_{0} s \hat{\alpha}+2 m_{0} s \alpha^{*}}(\hat{\xi})^{15 / 2}\left|g_{1}\right|^{2} d x d t \\
& +C s^{7} \lambda^{12} \int_{\omega_{1} \times(0, T)} e^{-2 s \alpha-2 a_{0} s \alpha^{*}} \xi^{7}|\nabla \times \varphi|^{2} d x d t
\end{aligned}
$$

for any $\lambda \geq C, s \geq C T^{10}$. 
On the other hand, considering open sets $\omega_{2}, \omega_{3} \Subset \omega$ such that $\omega_{1} \Subset \omega_{2} \Subset \omega_{3} \subset \omega$, we can deduce that

$$
\begin{aligned}
& s^{7} \lambda^{12} \iint_{\omega_{1} \times(0, T)} e^{-2 s \alpha-2 a_{0} s \alpha^{*}} \xi^{7}|\nabla \times \varphi|^{2} d x d t \\
& \leq C(\varepsilon) s^{15} \lambda^{24} \iint_{\omega_{3} \times(0, T)} e^{2\left(m_{0}-2\right) s \alpha^{*}-4 a_{0} s \alpha^{*}}(\hat{\xi})^{15}|\varphi|^{2} d x d t \\
& \quad+\varepsilon\left(\iint_{Q} e^{-2 m_{0} s \alpha}\left[s^{-1} \xi^{-1}|\Delta \varphi|^{2}+s \lambda^{2} \xi|\nabla \varphi|^{2}+s^{3} \lambda^{4} \xi^{3}|\varphi|^{2}\right] d x d t\right),
\end{aligned}
$$

for any $\lambda \geq C, s \geq C T^{10}$ and any $\varepsilon>0$.

Taking into account that $m_{0}>a_{0}+1$, there exists a constant $C>0$ such that

$$
s^{16} \lambda^{40} \iint_{\omega_{0} \times(0, T)} e^{-8 s m_{0} \hat{\alpha}+6 s m_{0} \alpha^{*}}(\hat{\xi})^{16}|\varphi|^{2} d x d t \leq C s^{15} \lambda^{24} \iint_{\omega_{3} \times(0, T)} e^{2\left(m_{0}-2\right) s \alpha^{*}-4 a_{0} s \alpha^{*}}(\hat{\xi})^{15}|\varphi|^{2} d x d t .
$$

From (3.23)-(3.25), we conclude the proof of Proposition 3.1.

\subsection{Null controllability of the linear system}

In this section we are concerned in the null controllability of the linear coupled Stokes system

$$
\begin{cases}y_{t}-\Delta y+\nabla p=f_{1}+h 1_{\omega}+\left(-\ell^{-2} \chi_{\mathcal{O}}+\gamma^{-2}\right) q & \text { in } \quad Q, \\ -z_{t}-\Delta z+\nabla \pi=f_{2}+\mu\left(y-y_{d}\right) \chi_{\mathcal{O}_{d}} & \text { in } Q, \\ \nabla \cdot y=0, \nabla \cdot z=0 & \text { in } \quad Q, \\ y=z=0 & \text { on } \Sigma, \\ y(\cdot, 0)=y_{0}(\cdot), \quad z(\cdot, T)=0 & \text { in } \Omega,\end{cases}
$$

where the functions $f_{1}$ and $f_{2}$ are in appropriate weighted spaces. We look for a control $h \in$ $L^{2}(\omega \times(0, T))^{N}$ such that, under suitable properties on $f_{1}, f_{2}$, the solution to (3.26) satisfies $y(\cdot, T)=0$ in $\Omega$.

To do this, let us first state a Carleman inequality with weight functions not vanishing in $t=0$. Let $\tilde{\ell} \in C^{1}([0, T])$ be a positive function in $[0, T)$ such that:

$$
\tilde{\ell}(t)=T^{2} / 4 \forall t \in[0, T / 2] \text { and } \tilde{\ell}(t)=t(T-t) \forall t \in[T / 2, T] .
$$

Now, we introduce the following weight functions

$$
\begin{aligned}
& \beta(x, t)=\frac{e^{12 \lambda\|\eta\|_{\infty}}-e^{\lambda\left(10\|\eta\|_{\infty}+\eta(x)\right)}}{\tilde{\ell}^{5}(t)}, \quad \tau(x, t)=\frac{e^{\lambda\left(10\|\eta\|_{\infty}+\eta(x)\right)}}{\tilde{\ell}^{5}(t)}, \\
& \beta^{*}(t)=\max _{x \in \bar{\Omega}} \beta(x, t), \quad \tau^{*}(t)=\min _{x \in \bar{\Omega}} \tau(x, t), \\
& \widehat{\beta}(t)=\min _{x \in \bar{\Omega}} \beta(x, t), \quad \widehat{\tau}(t)=\max _{x \in \bar{\Omega}} \tau(x, t) .
\end{aligned}
$$

Lemma 3.4. Let $s$ and $\lambda$ like in Proposition 3.1. Then, there exists a constant $C>0$ (depending 
on $s, \lambda, \omega, \mathcal{O}, T, \ell, \gamma$ and $\mu)$ such that every solution $(\varphi, \theta)$ of (3.2) satisfies

$$
\begin{gathered}
\|\varphi(\cdot, 0)\|_{L^{2}(Q)^{N}}^{2}+\iint_{Q} e^{-2 m_{0} s \beta^{*}}\left(\tau^{*}\right)^{3}|\varphi|^{2} d x d t+\iint_{Q} e^{-2\left(a_{0}+1\right) s \beta^{*}}\left(\tau^{*}\right)^{3}|\theta|^{2} d x d t \\
\leq C\left(\iint_{Q} e^{-2 a_{0} s \beta^{*}}(\hat{\tau})^{15}\left|g_{1}\right|^{2} d x d t+\iint_{Q} e^{-2 a_{0} s \beta^{*}}\left|g_{2}\right|^{2} d x d t\right. \\
\left.+\iint_{\omega \times(0, T)} e^{-4 a_{0} s \beta^{*}+2\left(m_{0}-2\right) s \beta}(\hat{\tau})^{15}|\varphi|^{2} d x d t\right) .
\end{gathered}
$$

Proof of Lemma 3.4. By construction $\alpha=\beta$ and $\xi=\tau$ in $\Omega \times(T / 2, T)$, so that

$$
\begin{aligned}
& \int_{T / 2}^{T} \int_{\Omega}\left(e^{-2\left(a_{0}+1\right) s \alpha^{*}}\left(\xi^{*}\right)^{3}|\theta|^{2}+e^{-2 s m_{0} \alpha^{*}}\left(\xi^{*}\right)^{3}|\varphi|^{2}\right) d x d t \\
& =\int_{T / 2}^{T} \int_{\Omega}\left(e^{-2\left(a_{0}+1\right) s \beta^{*}}\left(\tau^{*}\right)^{3}|\theta|^{2}+e^{-2 s m_{0} \beta^{*}}\left(\tau^{*}\right)^{3}|\varphi|^{2}\right) d x d t .
\end{aligned}
$$

Therefore, it follows from Proposition 3.1 the estimate

$$
\begin{aligned}
& \int_{T / 2}^{T} \int_{\Omega}\left(e^{-2\left(a_{0}+1\right) s \beta^{*}}\left(\tau^{*}\right)^{3}|\theta|^{2}+e^{-2 s m_{0} \beta^{*}}\left(\tau^{*}\right)^{3}|\varphi|^{2}\right) d x d t \\
& \leq C\left(\iint_{Q} e^{-2 a_{0} s \alpha^{*}}(\hat{\xi})^{5}\left|g_{1}\right|^{2} d x d t+\iint_{Q} e^{-2 a_{0} s \alpha^{*}}\left|g_{2}\right|^{2} d x d t\right. \\
& \left.+\iint_{\omega \times(0, T)} e^{-4 a_{0} s \alpha^{*}+2\left(m_{0}-2\right) s \alpha}(\hat{\xi})^{15}|\varphi|^{2} d x d t\right) .
\end{aligned}
$$

Since $\tilde{\ell}(t)=t(T-t)$ for any $t \in[T / 2, T]$ and

$$
e^{-2 a_{0} s \beta^{*}} \geq C, \quad e^{-2 a_{0} s \beta^{*}}\left(\tau^{*}\right)^{5} \geq C \quad \text { and } \quad e^{-4 a_{0} s \beta^{*}+2\left(m_{0}-2\right) s \beta}(\hat{\tau})^{15} \geq C \text { in }[0, T / 2],
$$

we readily get

$$
\begin{gathered}
\int_{T / 2}^{T} \int_{\Omega}\left(e^{-2\left(a_{0}+1\right) s \beta^{*}}\left(\tau^{*}\right)^{3}|\theta|^{2}+e^{-2 s m_{0} \beta^{*}}\left(\tau^{*}\right)^{3}|\varphi|^{2}\right) d x d t \\
\leq C\left(\iint_{Q} e^{-2 a_{0} s \beta^{*}}(\hat{\tau})^{5}\left|g_{1}\right|^{2} d x d t+\iint_{Q} e^{-2 a_{0} s \beta^{*}}\left|g_{2}\right|^{2} d x d t\right. \\
\left.+\iint_{\omega \times(0, T)} e^{-4 a_{0} s \beta^{*}+2\left(m_{0}-2\right) s \beta}(\hat{\tau})^{15}|\varphi|^{2} d x d t\right) .
\end{gathered}
$$

Now, we introduce a function $\nu \in C^{1}([0, T])$ such that $\nu \equiv 1$ in $[0, T / 2], \nu \equiv 0$ in $[3 T / 4, T]$. It is easy to see that $\left(\nu \varphi, \nu \pi_{\varphi}\right)$ and $\left(\nu \theta, \nu \pi_{\varphi}\right)$ satisfies the system

$$
\begin{cases}-(\nu \varphi)_{t}-\Delta(\nu \varphi)+\nabla\left(\nu \pi_{\varphi}\right)=\nu\left(g_{1}+\mu \theta \chi_{\mathcal{O}_{d}}\right)-\nu^{\prime} \varphi & \text { in } \quad Q, \\ (\nu \theta)_{t}-\Delta(\nu \theta)+\nabla\left(\nu \pi_{\theta}\right)=\nu\left(g_{2}-\ell^{-2} \varphi \chi_{\mathcal{O}}+\gamma^{-2} \varphi\right)+\nu^{\prime} \theta & \text { in } Q, \\ \nabla \cdot(\nu \varphi)=\nabla \cdot(\nu \theta)=0, \nabla \cdot q=0 & \text { in } \quad Q \\ \nu \varphi=\nu \theta=0 & \text { on } \Sigma, \\ (\nu \varphi)(T)=0, \quad \nu \theta(0)=0 & \text { in } \quad \Omega\end{cases}
$$


Using classical energy estimate for both $\nu \varphi$ and $\nu \theta$, which solve the Stokes system (3.30) we get

$$
\begin{aligned}
& \|\varphi(0)\|_{L^{2}(Q)^{N}}^{2}+\|\varphi\|_{L^{2}\left(0, T / 2 ; H_{0}^{1}(\Omega)^{N}\right)}^{2} \\
& \leq C\left(\frac{1}{T^{2}}\|\varphi\|_{L^{2}\left(T / 2, T / 4 ; L^{2}(\Omega)^{N}\right)}^{2}+\|\theta\|_{L^{2}\left(0,3 T / 4 ; L^{2}\left(\mathcal{O}_{d}\right)^{N}\right)}^{2}+\left\|g_{1}\right\|_{L^{2}\left(0,3 T / 2 ; L^{2}(\Omega)^{N}\right)}^{2}\right)
\end{aligned}
$$

and

$$
\begin{aligned}
\|\theta\|_{L^{2}\left(0, T / 2 ; H_{0}^{1}(\Omega)^{N}\right) \leq}^{2} \leq C & \frac{1}{T^{2}}\|\theta\|_{L^{2}\left(T / 2,3 T / 4 ; L^{2}(\Omega)^{N}\right)}^{2} \\
& \left.\quad\left\|\nu\left(-\ell^{-2} \varphi \chi_{\mathcal{O}}+\gamma^{-2} \varphi\right)\right\|_{L^{2}\left(0,3 T / 4 ; L^{2}(\Omega)^{N}\right)}^{2}+\left\|g_{2}\right\|_{L^{2}\left(0,3 T / 2 ; L^{2}(\Omega)^{N}\right)}^{2}\right) .
\end{aligned}
$$

Taking into account that

$$
e^{-2 s m_{0} \beta^{*}}\left(\tau^{*}\right)^{3} \geq C>0 \quad e^{-2\left(a_{0}+1\right) s \beta^{*}}\left(\tau^{*}\right)^{3} \geq C>0, \quad \forall t \in[T / 2,3 T / 4]
$$

and

$$
e^{-2 a_{0} s \beta^{*}}(\hat{\tau})^{5} \geq C>0 \quad e^{-2 a_{0} s \beta^{*}}>e^{-4 a_{0} s \beta^{*}} \geq C>0, \quad \forall t \in[0,3 T / 4]
$$

we have

$$
\begin{aligned}
& \|\varphi(0)\|_{L^{2}(\Omega)^{N}}^{2}+\int_{0}^{T / 2} \int_{\Omega} e^{-2 m_{0} s \beta^{*}}\left(\tau^{*}\right)^{3}|\varphi|^{2} d x d t+\int_{0}^{T / 2} \int_{\Omega} e^{-2\left(a_{0}+1\right) s \beta^{*}}\left(\tau^{*}\right)^{3}|\theta|^{2} d x d t \\
& \leq C\left(\int_{T / 2}^{3 T / 2} \int_{\Omega}\left[e^{-2 m_{0} s \beta^{*}}\left(\tau^{*}\right)^{3}|\varphi|^{2}+e^{-2\left(a_{0}+1\right) s \beta^{*}}\left(\tau^{*}\right)^{3}|\theta|^{2}\right] d x d t\right. \\
& \quad+\left\|\nu \mu e^{-2 a_{0} s \beta^{*}} \theta\right\|_{L^{2}\left(0,3 T / 4 ; L^{2}\left(\mathcal{O}_{d}\right)^{N}\right)}^{2}+\left\|\nu\left(-\ell^{-2} \varphi \chi_{\mathcal{O}}+\gamma^{-2} \varphi\right)\right\|_{L^{2}\left(0,3 T / 4 ; L^{2}(\Omega)^{N}\right)}^{2} \\
& \left.\quad+\int_{0}^{3 T / 4} \int_{\Omega}\left[e^{-2 a_{0} s \beta^{*}}\left(\tau^{*}\right)^{5}\left|g_{1}\right|^{2}+e^{-2 a_{0} s \beta^{*}}\left|g_{2}\right|^{2}\right] d x d t\right) .
\end{aligned}
$$

Thus, from (3.29) and (3.31) we have at this moment

$$
\begin{aligned}
& \|\varphi(0)\|_{L^{2}(\Omega)^{N}}^{2}+\iint_{Q}\left(e^{-2\left(a_{0}+1\right) s \beta^{*}}\left(\tau^{*}\right)^{3}|\theta|^{2}+e^{-2 s m_{0} \beta^{*}}\left(\tau^{*}\right)^{3}|\varphi|^{2}\right) d x d t \\
& \leq C\left(\iint_{Q} e^{-2 a_{0} s \beta^{*}}(\hat{\tau})^{5}\left|g_{1}\right|^{2} d x d t+\iint_{Q} e^{-2 a_{0} s \beta^{*}}\left|g_{2}\right|^{2} d x d t\right. \\
& \quad+\iint_{\omega \times(0, T)} e^{-4 a_{0} s \beta^{*}+2\left(m_{0}-2\right) s \beta}(\hat{\tau})^{15}|\varphi|^{2} d x d t \\
& \left.\quad+\left\|\nu \mu e^{-2 a_{0} s \beta^{*}} \theta\right\|_{L^{2}\left(0,3 T / 4 ; L^{2}\left(\mathcal{O}_{d}\right)^{N}\right)}^{2}+\left\|\nu\left(-\ell^{-2} \varphi \chi_{\mathcal{O}}+\gamma^{-2} \varphi\right)\right\|_{L^{2}\left(0,3 T / 4 ; L^{2}(\Omega)^{N}\right)}^{2}\right) .
\end{aligned}
$$

Observe that if $\ell$ and $\gamma$ are large enough, the last term in the right-hand side of (3.32) can be absorbed by the left-hand side. In addition, considering $\theta^{*}(x, t)=e^{-2 s \beta^{*}} \theta(x, t)$ instead $\nu \theta$ in (3.30) and using standard energy estimate for the system associated to $\theta$, we obtain

$$
\begin{aligned}
\int_{0}^{3 T / 4} \int_{\Omega} \nu^{2} \mu^{2} e^{-4 a_{0} s \beta^{*}}|\theta|^{2} d x d t \leq & C\left(\iint_{Q} e^{-4 a_{0} s \beta^{*}}\left|g_{2}\right|^{2} d x d t+\frac{1}{\ell^{4}} \int_{0}^{T} \int_{\mathcal{O}_{d}} e^{-4 a_{0} s \beta^{*}}|\varphi|^{2} d x d t\right. \\
& \left.+\frac{1}{\gamma^{4}} \iint_{Q} e^{-4 a_{0} s \beta^{*}}|\varphi|^{2} d x d t+\iint_{Q} e^{-4 a_{0} s \beta^{*}}\left(\tau^{*}\right)^{6 / 5}|\theta|^{2} d x d t\right) .
\end{aligned}
$$


Putting together (3.32), (3.33) and taking again $\ell$ and $\gamma$ large enough, we obtain the desired inequality (3.28).

Remark 3.2. In order to establish a null controllability result for the system (3.26) with suitable weight functions, see Theorem 3.1, observe that on the left-hand side of (3.4) it is possible to add the term

$$
\iint_{Q} e^{-4 a_{0} s \beta^{*}}\left(\tau^{*}\right)^{3}|\theta|^{2} d x d t
$$

This is a consequence of the inequalities $a_{0}+1<m_{0} \leq 2 a_{0}, a_{0} \geq \frac{5}{4}$.

Now, we are ready to prove the null controllability of system (3.26). The idea is to look a solution in an appropriate weighted functional space. Let us introduce the following space

$$
\begin{aligned}
& E:=\left\{\left(y, z, \pi_{y}, \pi_{z}, h\right): e^{a_{0} s \beta^{*}}(\hat{\tau})^{-5 / 2} y \in L^{2}(Q)^{N}, e^{a_{0} s \beta^{*}} z \in L^{2}(Q)^{N}\right. \text {, } \\
& e^{2 a_{0} s \beta^{*}-\left(m_{0}-2\right) s \hat{\beta}}(\hat{\tau})^{-15 / 2} h 1_{\omega} \in L^{2}(Q)^{N}, \\
& e^{a_{0} s \beta^{*}}(\hat{\tau})^{-15 / 2} y \in L^{2}\left(0, T ; H^{2}(\Omega)^{N}\right) \cap L^{\infty}(0, T ; V), \\
& e^{a_{0} s \beta^{*}}\left(\tau^{*}\right)^{-c_{0}} z \in L^{2}\left(0, T ; H^{2}(\Omega)^{N}\right) \cap L^{\infty}(0, T ; V), c_{0} \geq \frac{5}{2}, \\
& e^{m_{0} s \beta^{*}}\left(\hat{\tau}^{*}\right)^{-3 / 2}\left(y_{t}-\Delta y+\nabla \pi_{y}-\left(-\ell^{-2} \chi_{\mathcal{O}}+\gamma^{-2}\right) z-h 1_{\omega}\right) \in L^{2}(Q)^{N} \text {, } \\
& \left.e^{2 a_{0} s \beta^{*}}\left(\hat{\tau}^{*}\right)^{-3 / 2}\left(-z_{t}-\Delta z+\nabla \pi_{z}-\mu\left(y-y_{d}\right) \chi_{\mathcal{O}_{d}}\right) \in L^{2}(Q)^{N}\right\} \text {. }
\end{aligned}
$$

It is clear that $E$ is a Banach space for the following norm:

$$
\begin{aligned}
\left\|\left(y, z, \pi_{y}, \pi_{z}, h\right)\right\|_{E}:= & \left\|e^{a_{0} s \beta^{*}}(\hat{\tau})^{-5 / 2} y\right\|_{L^{2}(Q)^{N}}+\left\|e^{a_{0} s \beta^{*}} z\right\|_{L^{2}(Q)^{N}} \\
& +\| e^{2 a_{0} s \beta^{*}-\left(m_{0}-2\right) s \hat{\beta}(\hat{\tau})^{-15 / 2} h 1_{\omega} \|_{L^{2}(Q)^{N}}} \\
& +\left\|e^{a_{0} s \beta^{*}}(\hat{\tau})^{-15 / 2} y\right\|_{L^{2}\left(0, T ; H^{2}(\Omega)^{N}\right)}+\left\|e^{a_{0} s \beta^{*}}(\hat{\tau})^{-15 / 2} y\right\|_{L^{\infty}(0, T ; V)} \\
& +\left\|e^{a_{0} s \beta^{*}}\left(\tau^{*}\right)^{-c_{0}} z\right\|_{L^{2}\left(0, T ; H^{2}(\Omega)^{N}\right)}+\left\|e^{a_{0} s \beta^{*}}\left(\tau^{*}\right)^{-c_{0}} z\right\|_{L^{\infty}(0, T ; V)} \\
& +\left\|e^{m_{0} s \beta^{*}}\left(\tau^{*}\right)^{-3 / 2}\left(y_{t}-\Delta y+\nabla \pi_{y}-\left(-\ell^{-2} \chi_{\mathcal{O}}+\gamma^{-2}\right) z-h 1_{\omega}\right)\right\|_{L^{2}(Q)^{N}} \\
& +\left\|e^{2 a_{0} s \beta^{*}}\left(\tau^{*}\right)^{-3 / 2}\left(-z_{t}-\Delta z+\nabla \pi_{z}-\mu\left(y-y_{d}\right) \chi_{\mathcal{O}_{d}}\right)\right\|_{L^{2}(Q)^{N}} .
\end{aligned}
$$

Remark 3.3. Observe in particular that $\left(y, z, \pi_{y}, \pi_{z}, h\right) \in E$ implies $y(\cdot, T)=0$ in $\Omega$.

Theorem 3.1. Assume the hypothesis of Lemma 3.4 and

$$
y_{0} \in V, \quad e^{m_{0} s \beta^{*}}\left(\tau^{*}\right)^{-3 / 2} f_{1} \in L^{2}(Q)^{N}, \quad e^{2 a_{0} s \beta^{*}}\left(\tau^{*}\right)^{-3 / 2} f_{2} \in L^{2}(Q)^{N} .
$$

Then, we can find a control $h \in L^{2}(\omega \times(0, T))^{N}$ such that the associated solution $\left(y, z, \pi_{y}, \pi_{z}, h\right)$ to (3.26) satisfies $\left(y, z, \pi_{y}, \pi_{z}, h\right) \in E$.

Proof of Theorem 3.1. Let us introduce the following constrained extremal problem:

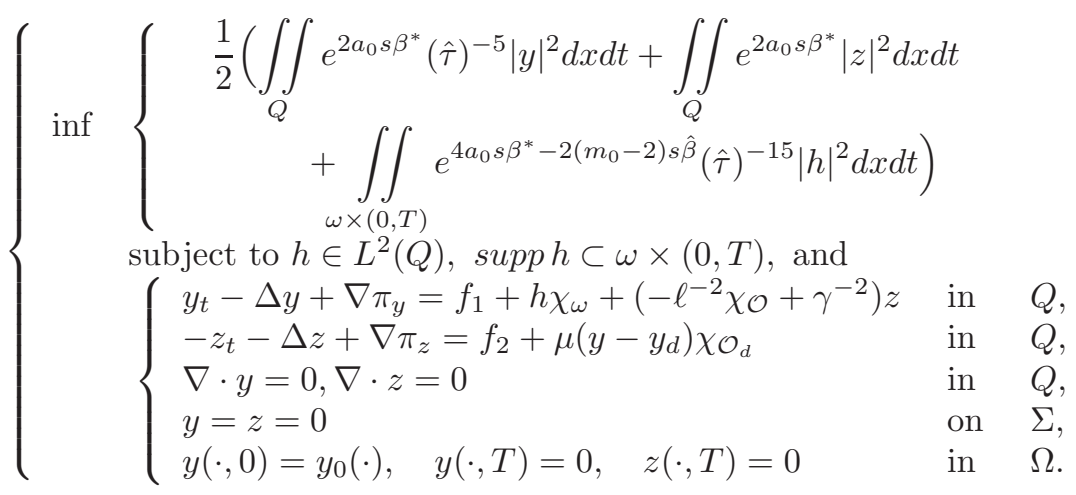


Assume that this problem admits a unique solution $\left(\hat{y}, \hat{z}, \hat{\pi}_{y}, \hat{\pi}_{z}, \hat{h}\right)$. Then, from the Lagrange's principle there exists dual variables $\left(\hat{\varphi}, \hat{\theta}, \hat{\pi}_{\varphi}, \hat{\pi}_{\theta}\right)$ such that

$$
\begin{array}{ll}
\hat{y}=e^{-2 a_{0} s \beta^{*}}(\hat{\tau})^{5}\left(-\hat{\varphi}_{t}-\Delta \hat{\varphi}+\nabla \hat{\pi}_{\varphi}-\mu \hat{\theta}_{\mathcal{O}_{d}}\right) & \text { in } \quad Q, \\
\hat{z}=e^{-2 a_{0} s \beta^{*}}\left(\hat{\theta}_{t}-\Delta \hat{\theta}+\nabla \hat{\pi}_{\theta}-\left(-\ell^{-2} \chi_{\mathcal{O}}+\gamma^{-2}\right) \hat{\varphi}\right) & \text { in } \quad Q, \\
\hat{h}=e^{-4 a_{0} s \beta^{*}+2\left(m_{0}-2\right) s \hat{\beta}}(\hat{\tau})^{15} \hat{\varphi} & \text { in } \quad Q, \\
\hat{y}=\hat{z}=0 & \text { on } \Sigma .
\end{array}
$$

Now, following the arguments established in [10], we introduce the space $P_{0}$ of functions $\left(y, z, \pi_{y}, \pi_{z}\right) \in C^{2}(\bar{Q})^{2 N+2}$ such that

i) $\nabla \cdot y=\nabla \cdot z=0$ in $Q$.

ii) $y=z=0$ on $\Sigma$.

iii) $\int_{\omega_{0}} \pi_{\varphi} d x=0$.

We also consider the bilinear form $a(\cdot, \cdot)$ over $P_{0} \times P_{0}$ defined by:

$$
\begin{aligned}
& a\left(\left(\hat{\varphi}, \hat{\theta}, \hat{\pi}_{\varphi}, \hat{\pi}_{\theta}\right),\left(w, z, \pi_{w}, \pi_{z}\right)\right)=: \\
& \iint_{Q} e^{-2 a_{0} s \beta^{*}}(\hat{\tau})^{5}\left(-\hat{\varphi}_{t}-\Delta \hat{\varphi}+\nabla \hat{\pi}_{\varphi}-\mu \hat{\theta}_{\mathcal{O}_{d}}\right)\left(-y_{t}-\Delta y+\nabla \pi_{y}-\mu z_{\mathcal{O}_{d}}\right) d x d t \\
& \quad+\iint_{Q} e^{-2 a_{0} s \beta^{*}}\left(\hat{\theta}_{t}-\Delta \hat{\theta}+\nabla \hat{\pi}_{\theta}-\left(-\ell^{-2} \chi_{\mathcal{O}}+\gamma^{-2}\right) \hat{\varphi}\right)\left(z_{t}-\Delta z+\nabla \pi_{z}\right) \\
& \left.\quad-\iint_{Q} e^{-2 a_{0} s \beta^{*}}\left(\hat{\theta}_{t}-\Delta \hat{\theta}+\nabla \hat{\pi}_{\theta}-\left(-\ell^{-2} \chi_{\mathcal{O}}+\gamma^{-2}\right) \hat{\varphi}\right)\left(\ell^{-2} \chi_{\mathcal{O}}+\gamma^{-2}\right) w\right) d x d t \\
& +\iint_{\omega \times(0, T)} e^{-4 a_{0} s \beta^{*}+2\left(m_{0}-2\right) s \hat{\beta}}(\hat{\tau})^{15} \hat{\varphi} w d x d t,
\end{aligned}
$$

for every $\left(w, z, \pi_{w}, \pi_{z}\right) \in P_{0}$, and a linear form

$$
\left\langle G,\left(w, z, \pi_{w}, \pi_{z}\right)\right\rangle:=\iint_{Q} f_{1} \cdot w d x d t+\iint_{Q} f_{2} \cdot z d x d t+\iint_{\Omega} y_{0}(\cdot) \cdot w(\cdot, 0) d x .
$$

Taking into account this definitions, one can see that, if the functions $\hat{y}, \hat{z}$ and $\hat{h}$ solve (3.36), we must have

$$
a\left(\left(\hat{\varphi}, \hat{\theta}, \hat{\pi}_{\varphi}, \hat{\pi}_{\theta}\right),\left(w, z, \pi_{w}, \pi_{z}\right)\right)=\left\langle G,\left(w, z, \pi_{w}, \pi_{z}\right)\right\rangle, \quad \forall\left(w, z, \pi_{w}, \pi_{z}\right) \in P_{0} .
$$

Observe that Carleman inequality (3.28) holds for all $\left(w, z, \pi_{w}, \pi_{z}\right) \in P_{0}$. Consequently,

$$
\begin{aligned}
& \iint_{Q} e^{-2 m_{0} s \beta^{*}}\left(\tau^{*}\right)^{3}|z|^{2} d x d t+\iint_{Q} e^{-2\left(a_{0}+1\right) s \beta^{*}}\left(\tau^{*}\right)^{3}|w|^{2} d x d t \\
& +\iint_{Q} e^{-2 a_{0} s \beta^{*}}\left(\tau^{*}\right)^{3}|w|^{2} d x d t+\|w(0)\|_{L^{2}(\Omega)^{N}}^{2} \leq C a\left(\left(w, z, \pi_{w}, \pi_{z}\right),\left(w, z, \pi_{w}, \pi_{z}\right)\right),
\end{aligned}
$$

for every $\left(w, z, \pi_{w}, \pi_{z}\right) \in P_{0}$.

Therefore, $a(\cdot, \cdot): P_{0} \times P_{0} \longmapsto \mathbb{R}$ is symmetric, definite positive bilinear form on $P_{0}$. We denote by $P$ the completion of $P_{0}$ for the norm induced by $a(\cdot, \cdot)$. Then, $a(\cdot, \cdot)$ is well-defined, continuous and again definite positive on $P$. Furthermore, in view of the Carleman inequality 
(3.28), the assumption (3.35) and (3.40), the linear form $\left(w, z, \pi_{w}, \pi_{z}\right) \longmapsto\left\langle G,\left(w, z, \pi_{w}, \pi_{z}\right)\right\rangle$ is well-defined and continuous on $P$. Indeed, for every $\left(w, z, \pi_{w}, \pi_{z}\right) \in P$,

$$
\begin{aligned}
\left\langle G,\left(w, z, \pi_{w}, \pi_{z}\right)\right\rangle \leq \leq & \left\|e^{\left(a_{0}+1\right) s \beta^{*}}\left(\tau^{*}\right)^{-3 / 2} f_{1}\right\|_{L^{2}(Q)^{N}}\left\|e^{-\left(a_{0}+1\right) s \beta^{*}}\left(\tau^{*}\right)^{3 / 2} w\right\|_{L^{2}(Q)^{N}} \\
& \quad+\left\|e^{m_{0} s \beta^{*}}\left(\tau^{*}\right)^{-3 / 2} f_{2}\right\|_{L^{2}(Q)^{N}}\left\|e^{-m_{0} s \beta^{*}}\left(\tau^{*}\right)^{3 / 2} z\right\|_{L^{2}(Q)^{N}}+\left\|y_{0}\right\|_{H}\|w(0)\|_{H} \\
\leq & \left\|e^{m_{0} s \beta^{*}}\left(\tau^{*}\right)^{-3 / 2} f_{1}\right\|_{L^{2}(Q)^{N}}\left\|e^{-\left(a_{0}+1\right) s \beta^{*}}\left(\tau^{*}\right)^{3 / 2} w\right\|_{L^{2}(Q)^{N}} \\
& \quad+\left\|e^{2 a_{0} s \beta^{*}}\left(\tau^{*}\right)^{-3 / 2} f_{2}\right\|_{L^{2}(Q)^{N}}\left\|e^{-m_{0} s \beta^{*}}\left(\tau^{*}\right)^{3 / 2} z\right\|_{L^{2}(Q)^{N}}+\left\|y_{0}\right\|_{H}\|w(0)\|_{H}
\end{aligned}
$$

Using (3.40) and the density of $P_{0}$ in $P$, we find

$$
\begin{aligned}
\left\langle G,\left(w, z, \pi_{w}, \pi_{z}\right)\right\rangle \leq & C\left(\left\|e^{m_{0} s \beta^{*}}\left(\tau^{*}\right)^{-3 / 2} f_{1}\right\|_{L^{2}(Q)^{N}}\right. \\
& \left.+\left\|e^{2 a_{0} s \beta^{*}}\left(\tau^{*}\right)^{-3 / 2} f_{2}\right\|_{L^{2}(Q)^{N}}+\left\|y_{0}\right\|_{H}\right)\left\|\left(w, z, \pi_{w}, \pi_{z}\right)\right\|_{P} .
\end{aligned}
$$

Hence, from Lax-Milgram's Lemma, there exists a unique $\left(\hat{\varphi}, \hat{\theta}, \hat{\pi}_{\varphi}, \hat{\pi}_{\theta}\right) \in P$ satisfying:

$$
a\left(\left(\hat{\varphi}, \hat{\theta}, \hat{\pi}_{\varphi}, \hat{\pi}_{\theta}\right),\left(w, z, \pi_{w}, \pi_{z}\right)\right)=\left\langle G,\left(w, z, \pi_{w}, \pi_{z}\right)\right\rangle, \quad \forall\left(w, z, \pi_{w}, \pi_{z}\right) \in P .
$$

Let us set $(\hat{y}, \hat{z}, \hat{h})$ like in $(3.37)$ and remark that $\left(\hat{y}, \hat{z}, \hat{\pi}_{y}, \hat{\pi}_{z}, \hat{h}\right)$ verifies

$$
\begin{aligned}
a\left(\left(\hat{\varphi}, \hat{\theta}, \hat{\pi}_{\varphi}, \hat{\pi}_{\theta}\right),\left(\hat{\varphi}, \hat{\theta}, \hat{\pi}_{\varphi}, \hat{\pi}_{\theta}\right)\right)= & \iint_{Q} e^{2 a_{0} s \beta^{*}}(\hat{\tau})^{-5}|\hat{y}|^{2} d x d t+\iint_{Q} e^{2 a_{0} s \beta^{*}}|\hat{z}|^{2} d x d t \\
& +\iint_{\omega \times(0, T)} e^{4 a_{0} s \beta^{*}-2\left(m_{0}-2\right) s \hat{\beta}}(\hat{\tau})^{-15}|\hat{h}| d x d t<+\infty .
\end{aligned}
$$

Let us prove that $(\hat{y}, \hat{z})$ is, together with some $\left(\hat{\pi}_{y}, \hat{\pi}_{z}\right)$, the weak solution of the Stokes system in (3.36) for $h=\hat{h}$. In fact, we introduce the (weak) solution $\left(\tilde{y}, \tilde{z}, \tilde{\pi}_{y}, \tilde{\pi}_{z}\right)$ to the Stokes system

$$
\left\{\begin{array}{lll}
\tilde{y}_{t}-\Delta \tilde{y}+\nabla \tilde{\pi}_{y}=f_{1}+\hat{h} 1_{\omega}+\left(-\ell^{-2} \chi_{\mathcal{O}}+\gamma^{-2}\right) \tilde{z} & \text { in } & Q, \\
-\tilde{z}_{t}-\Delta \tilde{z}+\nabla \tilde{\pi}_{z}=f_{2}+\mu\left(\tilde{y}-\tilde{y}_{d}\right) \chi_{\mathcal{O}_{d}} & \text { in } & Q \\
\nabla \cdot \tilde{y}=0, \nabla \cdot \tilde{z}=0 & \text { in } & Q \\
\tilde{y}=\tilde{z}=0 & \text { on } & \Sigma \\
\tilde{y}(\cdot, 0)=y_{0}(\cdot), \quad \tilde{z}(\cdot, T)=0 & \text { in } & \Omega
\end{array}\right.
$$

Clearly, $(\tilde{y}, \tilde{z})$ is the unique solution of $(3.42)$ defined by transposition. This means that, for every $(a, b) \in L^{2}(Q)^{2 N}$,

$$
\langle(\tilde{y}, \tilde{z}),(a, b)\rangle_{L^{2}(Q)^{N}}=\left\langle y_{0}, \varphi(0)\right\rangle_{L^{2}(\Omega)}+\left\langle\left(f_{1}+\hat{h} 1_{\omega}, f_{2}\right),(\varphi, \theta)\right\rangle_{L^{2}(Q)^{N}},
$$

where $(\varphi, \theta)$ is, together with some $\left(\pi_{\varphi}, \pi_{\theta}\right)$, the solution to

$$
\begin{cases}L^{*}(\varphi, \theta)=(a, b) & \text { in } Q \\ \nabla \cdot \varphi=0, \nabla \cdot \theta=0 & \text { in } Q \\ \varphi=\theta=0 & \text { on } \Sigma, \\ \varphi(\cdot, T)=0, \quad \theta(\cdot, 0)=0 & \text { in } \Omega\end{cases}
$$

and $L^{*}$ is the adjoint operator of $L$ given by:

$$
L(\tilde{y}, \tilde{z}):=\left(\tilde{y}_{t}-\Delta \tilde{y}+\nabla \tilde{\pi}_{y}-\left(-\ell^{-2} \chi_{\mathcal{O}}+\gamma^{-2}\right) \tilde{z},-\tilde{z}_{t}-\Delta \tilde{z}+\nabla \tilde{\pi}_{z}-\mu\left(\tilde{y}-\tilde{y}_{d}\right) \chi_{\mathcal{O}_{d}}\right) .
$$

From (3.37) and (3.39), we see that $(\hat{y}, \hat{z})$ also satisfies $(3.43)$. Consequently, $(\hat{y}, \hat{z})=(\tilde{y}, \tilde{z})$ and $(\hat{y}, \hat{z})$ is, together with some $\left(\hat{\pi}_{y}, \hat{\pi}_{z}\right)=\left(\tilde{\pi}_{y}, \tilde{\pi}_{z}\right)$, the weak solution to the system $(3.42)$.

Finally, we must see that $\left(\hat{y}, \hat{z}, \hat{\pi}_{y}, \hat{\pi}_{z}, \hat{h}\right) \in E$. We already know that

$$
e^{a_{0} s \beta^{*}}(\hat{\tau})^{-5 / 2} \hat{y}, e^{a_{0} s \beta^{*}} \hat{z}, \quad e^{2 a_{0} s \beta^{*}-\left(m_{0}-2\right) s \hat{\beta}}(\hat{\tau})^{-15 / 2} \hat{h} 1_{\omega} \in L^{2}(Q)^{N}
$$


and (see hypothesis (3.35))

$$
e^{m_{0} s \beta^{*}}\left(\tau^{*}\right)^{-3 / 2} f_{1} \in L^{2}(Q)^{N} \quad \text { and } \quad e^{2 a_{0} s \beta^{*}}\left(\tau^{*}\right)^{-3 / 2} f_{2} \in L^{2}(Q)^{N} .
$$

Thus, it only remains to check that

$$
e^{a_{0} s \beta^{*}}(\hat{\tau})^{-15 / 2} \hat{y}, e^{a_{0} s \beta^{*}}\left(\tau^{*}\right)^{-c_{0}} z \in L^{2}\left(0, T ; H^{2}(\Omega)^{N}\right) \cap L^{\infty}(0, T ; V),
$$

where $c_{0} \geq \frac{5}{2}$.

a) We define the functions

$$
\begin{array}{ll}
y^{*}:=e^{a_{0} s \beta^{*}}(\hat{\tau})^{-15 / 2} \hat{y}, & z^{*}:=e^{a_{0} s \beta^{*}}\left(\tau^{*}\right)^{-c_{0}} \hat{z} \\
\pi_{y}^{*}:=e^{a_{0} s \beta^{*}}(\hat{\tau})^{-15 / 2} \hat{\pi}_{y}, & \pi_{z}^{*}:=e^{a_{0} s \beta^{*}}\left(\tau^{*}\right)^{-c_{0}} \hat{\pi}_{z}
\end{array}
$$

and

$$
\begin{array}{ll}
f_{1}^{*}:=e^{a_{0} s \beta^{*}}(\hat{\tau})^{-15 / 2}\left(f_{1}+h 1_{\omega}\right), & z^{* *}:=e^{a_{0} s \beta^{*}}(\hat{\tau})^{-15 / 2}\left(-\ell^{-2} \chi_{\mathcal{O}}+\gamma^{-2}\right) z \\
f_{2}^{*}:=e^{a_{0} s \beta^{*}}\left(\tau^{*}\right)^{-c_{0}} f_{2}, & y^{* *}:=e^{a_{0} s \beta^{*}}\left(\tau^{*}\right)^{-c_{0}}\left(y-y_{d}\right) \chi_{\mathcal{O}_{d}} .
\end{array}
$$

Then $\left(y^{*}, \pi_{y}^{*}, z^{*}, \pi_{z}^{*}\right)$ satisfies:

$$
\left\{\begin{array}{lll}
y_{t}^{*}-\Delta y^{*}+\nabla \pi_{y}^{*}=f_{1}^{*}+z^{* *}+\left(e^{3 / 2 s \beta^{*}}(\hat{\tau})^{-15 / 2}\right)^{\prime} \hat{y} & \text { in } & Q, \\
-z_{t}^{*}-\Delta z^{*}+\nabla \pi_{z}^{*}=f_{2}^{*}+y^{* *}+\left(e^{1 / 2 s \beta^{*}}(\hat{\tau})^{7}\right)^{\prime} \hat{z} & \text { in } Q, \\
\nabla \cdot y^{*}=0, \nabla \cdot z^{*}=0 & \text { in } Q, \\
y^{*}=z^{*}=0 & \text { on } \Sigma, \\
y^{*}(\cdot, 0)=e^{3 / 2 s \beta^{*}(0)}(\hat{\tau}(0))^{-15 / 2} y_{0}(\cdot), \quad z^{*}(\cdot, T)=0 & \text { in } \Omega,
\end{array}\right.
$$

b) Now, we prove that the right-hand side of the main equations in (3.45) is in $L^{2}(Q)^{N}$.

- $\left|e^{a_{0} s \beta^{*}}(\hat{\tau})^{-15 / 2} f_{1}\right| \leq C e^{a_{0} s \beta^{*}}|\hat{\tau}|^{-15 / 2}\left|f_{1}\right| \leq C e^{m_{0} s \beta}\left|\tau^{*}\right|^{-3 / 2}\left|f_{1}\right|$.

- $\left|e^{a_{0} s \beta^{*}}(\hat{\tau})^{-15 / 2} h 1_{\omega}\right| \leq C e^{2 a_{0} s \beta^{*}-\left(m_{0}-2\right) s \beta^{*}}(\hat{\tau})^{-15 / 2}|h| 1_{\omega}$.

- $\left|z^{* *}\right|=\left|e^{a_{0} s \beta^{*}}(\hat{\tau})^{-15 / 2}\left(-\ell^{-2} \chi_{\mathcal{O}}+\gamma^{-2}\right) z\right| \leq C e^{a_{0} s \beta^{*}}|\hat{z}|$.

- $\left|\left(e^{3 / 2 s \beta^{*}}(\hat{\tau})^{-15 / 2}\right)^{\prime} \hat{y}\right| \leq C s e^{a_{0} s \beta^{*}}\left|\tau^{*}\right|^{6 / 5}|\hat{y}| \leq C e^{a_{0} s \beta^{*}}|\hat{\tau}|^{-5 / 2}|\hat{y}|$.

- $\left|f_{2}^{* *}\right|=\left|e^{a_{0} s \beta^{*}}\left(\tau^{*}\right)^{-c_{0}} f_{2}\right| \leq C e^{\left(a_{0}+1\right) s \beta^{*}}\left|\tau^{*}\right|^{-c_{0}}\left|f_{2}\right|$.

- $\left|\left(e^{1 / 2 s \beta^{*}}(\hat{\tau})^{7}\right)^{\prime} \hat{z}\right| \leq C e^{a_{0} s \beta^{*}}|\hat{z}|$.

- $\left|y^{* *}\right|=\left|e^{a_{0} s \beta^{*}}\left(\tau^{*}\right)^{-c_{0}}\left(y-y_{d}\right) \chi_{\mathcal{O}_{d}}\right| \leq C e^{a_{0} s \beta^{*}}|\hat{\tau}|^{-5 / 2}|\hat{y}|+C e^{a_{0} s \beta^{*}}\left|\tau^{*}\right|^{-c_{0}}\left|y_{d}\right|$.

Observe that $y^{* *} \in L^{2}(Q)^{N}$ thanks to the hypothesis (1.5).

Taking into account $a)-b$ ) and $y_{0} \in V$, we have $y^{*}, z^{*} \in L^{2}\left(0, T ; H^{2}(\Omega)^{N}\right) \cap L^{\infty}(0, T ; V)$ (see Lemma 3.3 in Section 3.1).

This concludes the proof of Proposition 3.1.

Remark 3.4. Before starting the last section, it is important to consider small data in order to prove our main result, Theorem 1.1. Thus, we impose that

$$
\left\|f_{1}\right\|_{L^{2}(Q)^{N}}+\left\|f_{2}\right\|_{L^{2}(Q)^{N}}+\left\|y_{0}\right\|_{V} \leq \delta,
$$

where $\delta$ is a small positive number.

\section{Proof of the main result}

In this section we give the proof of Theorem 1.1 throughout classical arguments such like in [10]. The results obtained in the previous section allow us to locally invert a nonlinear operator associated to the nonlinear system

$$
\begin{cases}y_{t}-\Delta y+(y \cdot \nabla) y+\nabla \pi_{y}=h 1_{\omega}+\left(\ell^{-2} \tilde{\chi}_{\mathcal{O}}+\gamma^{-2}\right) z & \text { in } \quad Q, \\ -z_{t}-\Delta z+\left(z, \nabla^{t}\right) y-(y, \nabla) z+\nabla \pi_{z}=\mu\left(y-y_{d}\right) \chi_{\mathcal{O}_{d}} & \text { in } \quad Q, \\ \nabla \cdot y=0, \nabla \cdot z=0 & \text { in } \quad Q \\ y=z=0 & \text { on } \quad \Sigma \\ y(\cdot, 0)=y_{0}(\cdot), \quad z(\cdot, T)=0 & \text { in } \quad \Omega .\end{cases}
$$


to do this, we will apply an inverse function theorem of the Luisternik's kind [16], which will allow us to complete the proof of theorem 1.1. More precisely, we will use the following theorem.

Theorem 4.1. Suppose that $\mathcal{B}_{1}, \mathcal{B}_{2}$ are Banach spaces and

$$
\mathcal{A}: \mathcal{B}_{1} \rightarrow \mathcal{B}_{2}
$$

is a continuously differentiable map. We assume that for $b_{1}^{0} \in \mathcal{B}_{1}, b_{2}^{0} \in \mathcal{B}_{2}$ the equality

$$
\mathcal{A}\left(b_{1}^{0}\right)=b_{2}^{0}
$$

holds and $\mathcal{A}^{\prime}\left(b_{1}^{0}\right): \mathcal{B}_{1} \rightarrow \mathcal{B}_{2}$ is an epimorphism. Then there exists $\delta>0$ such that for any $b_{2} \in \mathcal{B}_{2}$ which satisfies the condition

$$
\left\|b_{2}^{0}-b_{2}\right\|_{\mathcal{B}_{2}}<\delta
$$

there exists a solution $b_{1} \in \mathcal{B}_{1}$ of the equation

$$
\mathcal{A}\left(b_{1}\right)=b_{2} .
$$

We apply this theorem for the spaces $\mathcal{B}_{1}:=E$ and

$$
\mathcal{B}_{2}:=\left\{\left(f_{1}, f_{2}, y_{0}\right) \in X_{1}^{*} \times X_{2}^{*} \times V: f_{1}, f_{2}, y_{0} \text { satisfies }(3.46)\right\},
$$

where $X_{1}^{*}:=L^{2}\left(e^{m_{0} s \beta^{*}}\left(\tau^{*}\right)^{-3 / 2}(0, T) ; L^{2}(\Omega)^{N}\right)$ and $X_{2}^{*}:=L^{2}\left(e^{2 a_{0} s \beta^{*}}\left(\tau^{*}\right)^{-3 / 2}(0, T) ; L^{2}(\Omega)^{N}\right)$. We define the operator $\mathcal{A}$ by the formula

$$
\begin{aligned}
\mathcal{A}\left(y, z, \pi_{y}, \pi_{z}, h\right):=\left(y_{t}-\Delta y+(y \cdot \nabla) y+\nabla \pi_{y}-\left(\ell^{-2} \tilde{\chi}_{\mathcal{O}}+\gamma^{-2}\right) z-h 1_{\omega},\right. \\
\left.\quad-z_{t}-\Delta z+\left(z, \nabla^{t}\right) y-(y, \nabla) z+\nabla \pi_{z}-\mu\left(y-y_{d}\right) \chi_{\mathcal{O}_{d}}, y(\cdot, 0)\right),
\end{aligned}
$$

for every $\left(y, z, \pi_{y}, \pi_{z}, h\right) \in \mathcal{B}_{1}$.

Let us see that $\mathcal{A}$ is of class $C^{1}\left(\mathcal{B}_{1}, \mathcal{B}_{2}\right)$. Indeed, notice that all the terms in $\mathcal{A}$ are linear, except for $(y \cdot \nabla) y$ and $\left.z, \nabla^{t}\right) y-(y, \nabla) z$, then, we only have to check that these nonlinear terms are well-defined and depend continuously on the data. Thus, we will prove that the bilinear operator $\left(\left(y^{1}, z^{1}, \pi_{y}^{1}, \pi_{z}^{1}, h^{1}\right),\left(y^{2}, z^{2}, \pi_{y}^{2}, \pi_{z}^{2}, h^{2}\right)\right) \longmapsto\left(y^{1} \cdot \nabla\right) y^{2}$ is continuous from $\mathcal{B}_{1} \times \mathcal{B}_{1}$ to $X_{1}^{*}$, and the bilinear forms $\left(\left(y^{1}, z^{1}, \pi_{y}^{1}, \pi_{z}^{1}, h^{1}\right),\left(y^{2}, z^{2}, \pi_{y}^{2}, \pi_{z}^{2}, h^{2}\right)\right) \longmapsto\left(y^{1} \cdot \nabla\right) z^{2}$, $\left(\left(y^{1}, z^{1}, \pi_{y}^{1}, \pi_{z}^{1}, h^{1}\right),\left(y^{2}, z^{2}, \pi_{y}^{2}, \pi_{z}^{2}, h^{2}\right)\right) \longmapsto\left(z^{1} \cdot \nabla^{t}\right) y^{2}$ are continuous from $\mathcal{B}_{1} \times \mathcal{B}_{1}$ to $X_{2}^{*}$.

In fact, notice that (see the definition of the space $E$ ):

$$
e^{a_{0} s \beta^{*}}(\hat{\tau})^{-15 / 2} y \in L^{2}\left(0, T ; L^{\infty}(\Omega)^{N}\right)
$$

and

$$
\nabla\left(e^{a_{0} s \beta^{*}}(\hat{\tau})^{-15 / 2} y\right) \in L^{\infty}\left(0, T ; L^{2}(\Omega)^{N \times N}\right) .
$$

Consequently, we obtain

$$
\begin{aligned}
& \left\|e^{m_{0} s \beta^{*}}\left(\tau^{*}\right)^{-3 / 2}\left(y^{1} \cdot \nabla\right) y^{2}\right\|_{L^{2}(Q)^{N}} \\
& \leq C\left\|\left(e^{a_{0} s \beta^{*}}(\hat{\tau})^{-15 / 2} y^{1} \cdot \nabla\right) e^{a_{0} s \beta^{*}}(\hat{\tau})^{-15 / 2} y^{2}\right\|_{L^{2}(Q)^{N}} \\
& \leq C\left\|e^{2 s \beta^{*}}(\hat{\tau})^{-15 / 2} y^{1}\right\|_{L^{2}\left(0, T ; L^{\infty}(\Omega)^{N}\right)}\left\|e^{a_{0} s \beta^{*}}(\hat{\tau})^{-15 / 2} y^{2}\right\|_{L^{\infty}(0, T ; V)} .
\end{aligned}
$$

On the other hand, for $c_{0} \geq 5 / 2$,

$$
e^{a_{0} s \beta^{*}}\left(\tau^{*}\right)^{-c_{0}} z \in L^{2}\left(0, T ; L^{\infty}(\Omega)^{N}\right)
$$

and

$$
\nabla\left(e^{a_{0} s \beta^{*}}\left(\tau^{*}\right)^{-c_{0}} z\right) \in L^{\infty}\left(0, T ; L^{2}(\Omega)^{N \times N}\right) .
$$


Then,

$$
\begin{aligned}
& \left\|e^{2 a_{0} s \beta^{*}}\left(\tau^{*}\right)^{-3 / 2}\left(y^{1} \cdot \nabla\right) z^{2}\right\|_{L^{2}(Q)^{N}} \\
& \leq C\left\|e^{a_{0} s \beta^{*}}(\hat{\tau})^{-15 / 2} y^{1}\right\|_{L^{2}\left(0, T ; L^{\infty}(\Omega)^{N}\right)}\left\|e^{a_{0} s \beta^{*}}\left(\tau^{*}\right)^{-c_{0}} z^{2}\right\|_{L^{\infty}(0, T ; V)},
\end{aligned}
$$

and analogously,

$$
\begin{aligned}
& \left\|e^{2 a_{0} s \beta^{*}}\left(\tau^{*}\right)^{-3 / 2}\left(z^{1} \cdot \nabla\right) y^{2}\right\|_{L^{2}(Q)^{N}} \\
& \leq C\left\|e^{a_{0} s \beta^{*}}\left(\tau^{*}\right)^{-c_{0}} z^{1}\right\|_{L^{2}\left(0, T ; L^{\infty}(\Omega)^{N}\right)}\left\|e^{a_{0} s \beta^{*}}(\hat{\tau})^{-15 / 2} y^{2}\right\|_{L^{\infty}(0, T ; V)} .
\end{aligned}
$$

Notice that $\mathcal{A}^{\prime}(0,0,0): \mathcal{B}_{1} \rightarrow \mathcal{B}_{2}$ is given by

$$
\left(y_{t}-\Delta y+\nabla \pi_{y}-\left(\ell^{-2} \tilde{\chi}_{\mathcal{O}}+\gamma^{-2}\right) z-h 1_{\omega},-z_{t}-\Delta z+\nabla \pi_{z}-\mu\left(y-y_{d}\right) \chi_{\mathcal{O}_{d}}, y(\cdot, 0)\right),
$$

for all $\left(y, z, \pi_{y}, \pi_{z}, h\right) \in \mathcal{B}_{1}$.

In virtue of Theorem 3.1, this functional satisfies $\operatorname{Im}\left(\mathcal{A}^{\prime}(0,0,0)\right)=\mathcal{B}_{2}$.

Let $b_{1}^{0}=(0,0,0)$ and $b_{2}^{0}=(0,0)$. Then equation (4.1) obviously holds. So all necessary conditions to apply Theorem 4.1 are fulfilled. Therefore there exists a positive number $\delta$ such that, if $\|y(\cdot, 0)\|_{V} \leq \delta$, we can find a control $h \in L^{2}(\omega \times(0, T))^{N}$ and an associated solution $\left(y, z, \pi_{y}, \pi_{z}\right)$ to $(1.1)$ satisfying $y(\cdot, T)=0$ in $\Omega$. This finishes the proof of Theorem 1.1.

\section{Conclusion and open problems}

In this article, we mentioned the main results on robust control for the $N$-dimensional NavierStokes system with Dirichlet boundary conditions. These results has also allowed us to characterise the follower control $v$ and its disturbance function $\psi$ through a nonlinear coupled system. Once this step has finished, we used the robust pair $(v, \psi)$ to prove the null controllability of the leader control $h$. The main novelties are the Carleman inequalities for coupled Stokes system, which involves new relationships between the weight functions and the robustness parameters $\ell, \gamma$, see Proposition (3.1) and Lemma 6.1. To conclude, we present now some open problems arising from our study:

- If instead of considering in the hierarchical strategy a zero objective for the leader control $h$ in (1.1), the objective may be a trajectory $(\bar{y}, \bar{\pi})$ of the uncontrolled system:

$$
\begin{cases}\bar{y}_{t}-\Delta \bar{y}+(\bar{y} \cdot \nabla) \bar{y}+\nabla \bar{\pi}=0 & \text { in } Q \\ \nabla \cdot \bar{y}=0, & \text { in } Q \\ \bar{y}=0 & \text { on } \Sigma \\ \bar{y}(\cdot, 0)=\bar{y}_{0}(\cdot) & \text { in } \Omega\end{cases}
$$

So we may ask if is it possible to prove the local exact controllability to trajectories of system (1.1). That is, does there exist a control $h$ such that for the corresponding solution to $(2.5)$ satisfies $y(T)=\bar{y}(T)$ ?

- Some null controllability results for the $N$-dimensional Navier-Stokes equation ([?], [?]) allow to act on the system by means of few controls. Is it possible to extend these results to a robust Stackelberg strategy? Is is possible to ask the leader control $h$ to have one vanishing component?

- Is it possible to extend the results in this paper to Navier-slip boundary conditions?. In other words, can we say something about the existence and uniqueness of saddle points for the Navier-Stokes system with Navier-slip conditions? Does we have the null controllability for the leader control $h$ ?

- Finally, it would be interesting to study the problems proposed in this paper to other models such as water waves (Korteweg-de Vries equation), interaction fluid-heat (Boussinesq system), micropolar fluids, models of turbulence, among others. 


\section{Appendix: some technical results}

In the following results, it will be assumed that $N=2$ or $N=3$.

From the relation between $\alpha^{*}$ and $\hat{\alpha}$, it is possible to prove the following inequality.

Lemma 6.1. For any $\varepsilon>0$, any $M_{1}, M_{2} \in \mathbb{R}$, there exists $\lambda_{0}>0$ and $C=C\left(\varepsilon, M_{1}, M_{2}\right)>0$ such that

$$
e^{s \alpha^{*}} \leq C s^{M_{1}} \lambda^{M_{2}}(\hat{\xi})^{M_{1}} e^{s(1+\varepsilon) \hat{\alpha}}
$$

for every $\lambda>\lambda_{0}$.

Proof of Lemma 6.1. Recall that

$$
\alpha^{*}(t):=\max _{x \in \bar{\Omega}} \alpha(x, t), \quad \widehat{\alpha}(t):=\min _{x \in \bar{\Omega}} \alpha(x, t) \quad \text { and } \quad \widehat{\xi}(t):=\max _{x \in \bar{\Omega}} \xi(x, t) .
$$

From the definition of $\alpha^{*}$ and $\widehat{\alpha}, \widehat{\alpha}(t)=F(\lambda) \alpha^{*}$, where $F(\lambda):=\frac{e^{2 \lambda\left\|\eta_{0}\right\|_{\infty}}-e^{\lambda\left\|\eta_{0}\right\|_{\infty}}}{e^{2 \lambda\left\|\eta_{0}\right\|_{\infty}}-1}$. It is easy to check that $F(\lambda) \rightarrow 1$ to $\lambda \rightarrow+\infty$ and $F(\lambda) \rightarrow 1 / 2$ to $\lambda \rightarrow 0^{+}$. Additionally, by construction of $F(\lambda)$, for any $\varepsilon>0$, there exists $\lambda_{0}>0$ such that, for every $\lambda \geq \lambda_{0}$

$$
F(\lambda)+\varepsilon F(\lambda)>1
$$

In consequence, exists a positive constant $C=C\left(\varepsilon, M_{1}, \tilde{M}_{2}\right)$ such that the inequality

$$
\lambda^{\tilde{M}_{2}} e^{(1-(1+\varepsilon) F(\lambda)) s \alpha^{*}} \leq C s^{M_{1}}(\hat{\xi})^{M_{1}}
$$

holds for any $M_{1}, \tilde{M}_{2} \in \mathbb{R}$.

This completes the proof of Lemma 6.1.

As a consequence of Lemma 6.1, for $a_{0} \geq 2$ and $m_{0}$ satisfying $a_{0}<m_{0} \leq a_{0}+2$, we can deduce the next result:

Lemma 6.2. Under the hypothesis of Lemma 6.1, for any $\omega \Subset \Omega$ and any $u \in V$, there exists $\lambda_{0}>0$ and $C=C\left(\varepsilon, \tilde{M}_{1}, \tilde{M}_{2}\right)>0$ such that

$$
s^{\tilde{M}_{1}} \lambda^{\tilde{M}_{2}} \iint_{\omega \times(0, T)} e^{-4 s \hat{\alpha}-2 a_{0} s \alpha^{*}}(\hat{\xi})^{\tilde{M}_{1}}|\Delta u|^{2} d x d t \leq C s^{-1} \iint_{Q} e^{-2 m_{0} s \alpha^{*}}(\hat{\xi})^{-1}|\Delta u|^{2} d x d t .
$$

Sketch of the proof. Taking $\varepsilon=\frac{2}{m_{0}-a_{0}}-1, M_{1}=-\frac{\tilde{M}_{1}+1}{2\left(m_{0}-a_{0}\right)}$ and $M_{2}=-\frac{\tilde{M}_{2}+1}{2\left(m_{0}-a_{0}\right)}$ in (6.1), the proof is direct.

\section{References}

[1] F. D. Araruna, S.D.B. de Menezes, and M.A. Rojas-Medar. On the approximate controllability of stackelberg-nash strategies for linearized micropolar fluids. Applied Mathematics E) Optimization, 70(3):373-393, 2014.

[2] F.D. Araruna, E. Fernández-Cara, and MC Santos. Stackelberg-nash exact controllability for linear and semilinear parabolic equations. ESAIM: Control, Optimisation and Calculus of Variations, 21(3):835-856, 2015.

[3] F. Abergel and R. Temam. On some control problems in fluid mechanics. Theoretical and Computational Fluid Dynamics, 1(6):303-325, 1990. 
[4] T. R. Bewley, P. Moin, and R.Temam. Optimal and robust approaches for linear and nonlinear regulation problems in fluid mechanics. AIAA Paper, (97-1872), 1997.

[5] T. Bewley, R. Temam, and M. Ziane. Existence and uniqueness of optimal control to the navier-stokes equations. Comptes Rendus de l'Académie des Sciences-Series IMathematics, 330(11):1007-1011, 2000.

[6] T. R. Bewley, R. Temam, and M. Ziane. A general framework for robust control in fluid mechanics. Physica D: Nonlinear Phenomena, 138(3):360-392, 2000.

[7] J.I. Díaz and J.L. Lions. On the approximate controllability of stackelberg-nash strategies. In Ocean circulation and pollution control - a mathematical and numerical investigation, pages 17-27. Springer, 2004.

[8] I. Ekeland and R. Témam. Convex Analysis and Variational Problems, volume 28. SIAM, 1999.

[9] E. Fernández-Cara, M. González-Burgos, Sergio Guerrero, and J.-P. Puel. Null controllability of the heat equation with boundary fourier conditions: the linear case. ESAIM: Control, Optimisation and Calculus of Variations, 12(3):442-465, 2006.

[10] E. Fernández-Cara, S. Guerrero, O. Yu Imanuvilov, and J.-P. Puel. Local exact controllability of the navier-stokes system. Journal de mathématiques pures et appliquées, 83(12):1501-1542, 2004.

[11] A. V. Fursikov and O. Yu Imanuvilov. Controllability of evolution equations. Number 34. Seoul National University, 1996.

[12] F. Guillén-González, F. Marques-Lopes, and M. Rojas-Medar. On the approximate controllability of stackelberg-nash strategies for stokes equations. Proceedings of the American Mathematical Society, 141(5):1759-1773, 2013.

[13] M. Green and D. J.N. Limebeer. Linear robust control. Courier Corporation, 2012.

[14] S. Guerrero. Controllability of systems of stokes equations with one control force: existence of insensitizing controls. In Annales de l'IHP Analyse non linéaire, volume 24, pages 1029 1054, 2007.

[15] M. Gueye. Insensitizing controls for the navier-stokes equations. Annales de l'Institut Henri Poincaré (C) Non Linear Analysis, 30(5):825-844, 2013.

[16] R. S. Hamilton. The inverse function theorem of nash and moser. American Mathematical Society, 7(1):65-122, 1982.

[17] R. Herzog and F. Schmidt. Weak lower semi-continuity of the optimal value function and applications to worst-case robust optimal control problems. Optimization, 61(6):685-697, 2012.

[18] V. Hernández-Santamaría and L. de Teresa. Robust stackelberg controllability for a parabolic equation. arXiv preprint arXiv:1610.06149, 2016.

[19] J. Limaco, H.R. Clark, and L.A. Medeiros. Remarks on hierarchic control. Journal of Mathematical Analysis and Applications, 359(1):368-383, 2009.

[20] T. T. Medjo, R. Temam, and M. Ziane. Optimal and robust control of fluid flows: some theoretical and computational aspects. Applied Mechanics Reviews, 61(1):010802, 2008.

[21] R. Temam. Navier-Stokes equations: theory and numerical analysis, volume 343. American Mathematical Soc., 2001.

[22] H. Von Stackelberg. The theory of the market economy. Oxford University Press, 1952.

[23] C. Zalinescu. Convex analysis in general vector spaces. World scientific, 2002. 